\title{
SUR L'ALGÈBRE DES AUTOMORPHISMES INFINITÉSIMAUX D'UNE VARIÉTÉ SYMPLECTIQUE
}

\author{
ANDRÉ AVEZ, ANDRÉ LICHNEROWICZ \& A. DIAZ-MIRANDA
}

\section{INTRODUCTION}

On sait le rôle joué par les structures symplectiques en géométrie différentielle, comme en dynamique analytique (classique ou quantique). Nous nous proposons ici d'étudier la structure de l'algèbre de Lie $L$ des automorphismes infinitésimaux d'une variété symplectique $(W, F)$, algèbre de Lie à laquelle $\mathrm{E}$. Calabi s'est récemment intéressé. On sait que $L$ est de dimension infinie. L'algèbre de Lie des champs de vecteurs d'une variété différentiable, celle des vecteurs laissant invariante une forme élément de volume, l'algèbre de Lie des automorphismes infinitésimaux d'une structure de contact fournissent d'autres exemples naturels d'algèbres de Lie de dimension infinie. Ces algèbres ont fait récemment l'objet de travaux de Guelfand, Arnold et Rozenfeld dans le cas où la variété est compacte, de l'un d'entre nous sans cette hypothèse.

Les résultats principaux de ce travail apparaissent dans les parties III, IV et V. Dans un but de simplicité, on a regroupé dans les deux premières parties les notations, formules et rappels utilisés. L'un des principaux instruments est fourni par une généralisation d'un lemme de Calabi (§11).

La partie III porte principalement sur les algèbres de Lie admettant un produit scalaire invariant et on y établit des théorèmes de réductivité concernant certaines sous-algèbres de $L$. La partie IV est consacrée à l'étude des idéaux de l'algèbre de Lie $L$ ou de certains de ces idéaux. On montre en particulier que tous ces idéaux sont semi-simples et de dimension infinie, mais qu'aucun idéal $\neq\{0\}$ n'admet un idéal supplémentaire.

La partie $\mathrm{V}$ est relative à la détermination complète des dérivations de l'algèbre de Lie $L$, de son idéal $L^{*}$ correspondant aux 1 -formes exactes et de l'algèbre de Lie $N$ définie sur l'espace des fonctions $C^{\infty}$ à valeurs réelles par la parenthèse de Poisson. On en déduit la cohomologie de ces algèbres de Lie en dimension 1. L'algèbre de Lie $L^{c}$ des transformations infinitésimales conformes symplectiques joue ici un rôle essentiel. Si $W$ est compacte, les dérivations de $N$ ne sont pas toutes locales.

Communicated October 16, 1972. 
Certaines résultats ont été annoncés dans des notes par l'un ou l'autre d'entre nous.

\section{GÉNÉRALITÉS}

\section{Notion de variété symplectique}

a) Soit $W$ une variété différentiable connexe, paracompacte, de dimension $2 n ; T W$ (resp. $T^{*} W$ ) est le fibré des vecteurs (resp. covecteurs) tangents à $W$. Tous les éléments introduits sont supposés de classe $C^{\infty}$. Nous notons $N$ l'espace des fonctions $C^{\infty}(W, R), N_{0}$ le sous espace de $N$ des fonctions à supports compacts. Une structure symplectique est définie sur $W$ par une 2-forme $F$ fermée partout de rang $2 n$.

Nous notons $\left\{x^{i}\right\}(i$, tout indice latin $=1, \cdots, 2 n)$ une carte locale de $W$ de domaine $U$. De considérations locales chassiques, il résulte que $W$ admet des atlas de cartes locales dites canoniques $\left\{x^{\alpha}, x^{\bar{\alpha}}\right\}(\alpha=1, \cdots, n ; \bar{\alpha}=\alpha+n)$ telles que $F$ puisse s'écrire sur le domaine $U$ d'une de ces cartes:

$$
\left.F\right|_{U}=\sum_{\alpha} d x^{\alpha} \wedge d x^{\bar{\alpha}}
$$

Une structure symplectique est-au sens des $G$-structures-une $S p(n, R)$ structure intégrable; $\operatorname{Sp}(n, R)$ désigne ici le groupe symplectique réel à $2 n$ variables.

Sur la variété symplectique $(W, F)$ soit $\mu: T W \rightarrow T^{*} W$ l'isomorphisme de fibrés défini par $X \rightarrow-i(X) F$, où $i(\quad)$ désigne le produit intérieur. Cet isomorphisme s'étend naturellement aux fibrés de tenseurs; en ce qui concerne les tenseurs antisymétriques, il est compatible avec le produit extérieur. Pour deux $p$-formes $\alpha, \beta$, on a $i\left(\mu^{-1}(\alpha)\right) \beta=(-1)^{p} i\left(\mu^{-1}(\beta)\right) \alpha$.

b) Sur la variété symplectique $(W, F)$ la $2 n$-forme $\eta=F^{n} / n$ ! définit un élément de volume, l'élément de volume symplectique et la variété $W$ admet une orientation. Nous notons $N_{1}$ le sous espace de $N_{0}$ défini par les fonctions $u$ à supports compacts telles que:

$$
\int_{W} u \eta=0
$$

Si $W$ est compacte, l'élément de volume symplectique ne peut être une forme exacte; par suite la forme fermée $F$ (et ses puissances) ne peut être une forme exacte.

Nous notons $*$ l'opérateur d'adjonction symplectique sur les formes qui à toute $p$-forme $\alpha$ fait correspondre la $(2 n-p)$-forme:

$$
* \alpha=i\left(\mu^{-1}(\alpha)\right) \eta
$$


Si $\alpha$ et $\beta$ sont deux formes arbitraires, on a:

$$
*(\alpha \wedge \beta)=i\left(\mu^{-1}(\alpha \wedge \beta)\right) \eta=i\left(\mu^{-1}(\alpha) \wedge \mu^{-1}(\beta)\right)=i\left(\mu^{-1}(\beta)\right) i\left(\mu^{-1}(\alpha)\right) \eta .
$$

Ainsi

$$
*(\alpha \wedge \beta)=i\left(\mu^{-1}(\beta)\right)(* \alpha)
$$

On vérifie facilement que:

$$
*^{2}=\mathrm{Id}
$$

Sur $(W, F)$, nous disposons aussi de l'opérateur $L$ de degré 2 sur les formes, défini par le produit extérieur par $F$. De $L$ on déduit, à l'aide de $*$, l'opérateur de degré -2 :

$$
\Lambda=*^{-1} L *=* L *
$$

D’après (1.4), on a:

$$
\Lambda \alpha=*(F \wedge * \alpha)=i\left(\mu^{-1}(F)\right)\left(*^{2} \alpha\right) .
$$

Ainsi:

$$
\Lambda=i\left(\mu^{-1}(F)\right) .
$$

c) De l'opérateur $d$ de différentiation extérieure sur les formes, on déduit l'opérateur $\delta$ de codifférentiation symplectique défini de la manière suivante: si $\alpha$ est une $p$-forme:

$$
\delta \alpha=(-1)^{p} *^{-1} d * \alpha .
$$

On a manifestement $\delta^{2}=0$. On a [8] les formules suivantes:

(a) $d \Lambda-\Lambda d=-\delta$,

(b) $\delta L-L \delta=-d$.

Nous utiliserons exclusivement ici (1.8) (a) pour une 1-forme.

\section{Structure presque kählerienne subordonnée}

a) Sur la variété $(W, F)$, on sait [8] qu'il existe des métriques $g$ échangeables avec $F$, c'est-à-dire telles que $\mu^{-1}(g)$ soit le tenseur contravariant inverse de $g$. Désignons par $\nu: X \rightarrow i(X) g$ la dualité définie par la métrique $g$. Le produit $\mu^{-1} \circ \nu$ détermine sur $W$ un opérateur $\mathscr{J}$ de structure presque complexe. On obtient ainsi sur $W$ une structure $(W, F, g)$ dite presque kählerienne subordonnée à la structure symplectique envisagée.

On notera que l'élément de volume défini par $g$ coïncide avec $\eta$ et que $\nu^{-1}(F)=\mu^{-1}(F)$. La métrique $g$ définit un opérateur d'adjonction métrique $\tilde{*}$ 
sur les formes, qui vérifie pour une variété de dimension paire la relation

$$
\tilde{*}^{2}=(-1)^{p} \mathrm{Id} \text {. }
$$

Pour une variété presque hermitienne, on a:

$$
\tilde{*}^{-1} L \tilde{*}=i\left(\nu^{-1}(F)\right) \text {. }
$$

Comme $\nu^{-1}(F)=\mu^{-1}(F)$, il vient:

$$
\Lambda=\tilde{*}^{-1} L \tilde{*} .
$$

L'opérateur de structure presque complexe $\mathscr{J}$ définit sur les $p$-formes un opérateur $C$ par la relation:

$$
C \alpha\left(X_{(1)}, \cdots, X_{(p)}\right)=\alpha\left(\mathscr{J} X_{(1)}, \cdots, \mathscr{J} X_{(p)}\right),
$$

où $X_{(1)}, \cdots, X_{(p)}$ sont des vecteurs arbitraires. $C$ commute avec $\tilde{*}$ et $L$ et l'on a:

$$
C^{2}=(-1)^{p} \mathrm{Id}
$$

L'opérateur d'adjonction symplectique * peut s'exprimer, à partir de $*$ et $C$, par la relation

$$
*=\tilde{*} C^{-1} \text {. }
$$

On note que cette relation entraîne:

$$
*^{2}=\tilde{*} C^{-1} \tilde{*} C^{-1}=\tilde{*}^{2} C^{-2}=\mathrm{Id},
$$

c'est-à-dire (1.5).

b) Sur un domaine $U$ convenable de $W$, on peut écrire:

$$
\left.g\right|_{U}=2 \sum_{\alpha} \theta^{\alpha} \otimes \theta^{\bar{\alpha}},\left.\quad F\right|_{U}=i \sum_{\alpha} \theta^{\alpha} \wedge \theta^{\bar{\alpha}},
$$

où $\left(\theta^{\alpha}, \theta^{\alpha}=\bar{\theta}^{\alpha}\right)$ est un système adapté de formes de Pfaff à valeurs complexes. D'une manière générale, nous décomposons les tenseurs selon les types définis par l'opérateur $\mathscr{J}$ de structure presque complexe.

Soit $\nabla$ l'opérateur de dérivation covariante dans la connexion riemannienne définie par la métrique. Le tenseur $\nabla F$ relatif à toute structure presque hermitienne est tel que:

$$
\nabla_{k} F_{\alpha \bar{\beta}}=0 .
$$

D'autre part de $d F=0$, il résulte:

$$
(d F)_{\bar{\tau} \alpha \beta}=\nabla_{\bar{\gamma}} F_{\alpha \beta}+\nabla_{\alpha} F_{\beta \bar{\gamma}}+\nabla_{\beta} F_{\bar{\gamma} \alpha}=0
$$


et ainsi:

$$
\nabla_{\bar{\gamma}} F_{\alpha \beta}=0 \text {. }
$$

Soit $\bar{\delta}$ l'opérateur de codifférentiation métrique sur les formes. Si $\alpha$ est une $p$-forme:

$$
\bar{\delta} \alpha=(-1)^{p} \tilde{*}^{-1} d \tilde{*} \alpha
$$

Localement

$$
(\tilde{\delta} \alpha)_{j_{1} \ldots j_{p-1}}=-\nabla^{r} \alpha_{r j_{1} \cdots j_{p-1}}
$$

En particulier:

$$
(\tilde{\delta} F)_{\beta}=-\nabla^{r} F_{r \beta}=-\nabla^{\rho} F_{\rho \beta}-\nabla^{\rho} F_{\rho \beta}=0 .
$$

Ainsi sur une variété munie d'une structure presque kählerienne la forme fondamentale $F$ est à la fois fermée et cofermée par rapport à la métrique [8].

On peut évaluer $\delta$ en fonction de $\tilde{\delta}$ et $C$. Comme $*=\tilde{*} C^{-1}$, il vient :

$$
\delta=(-1)^{p} *^{-1} d *=(-1)^{p} C \tilde{*}^{-1} d \tilde{*} C^{-1} \alpha
$$

soit

$$
\delta=C \tilde{\delta} C^{-1}
$$

Ainsi, d'après (2.3),

$$
\delta=-C^{-1} \tilde{\delta} C
$$

La formule (1.8) (a) se réduit pour une 1-forme $\xi$ à :

$$
\Lambda d \xi=\delta \xi .
$$

Il est aisé de la vérifier en utilisant une structure presque kählerienne auxiliaire subordonnée à la structure symplectique. On a localement:

$$
\Lambda d \xi=\frac{1}{2} F^{i j}\left(\nabla_{i} \xi_{j}-\nabla_{j} \xi_{i}\right)=F^{i j} \nabla_{i} \xi_{j} .
$$

Soit $F$ étant cofermée.

$$
\Lambda d \xi=\nabla_{i}\left(F^{i j} \xi_{j}\right)
$$

D'autre part:

$$
\delta \xi=-\tilde{\delta} C \xi=\nabla^{i}\left(F_{i}{ }^{j} \xi_{j}\right)=\nabla_{i}\left(F^{i j} \xi_{j}\right),
$$

ce qui démontre (2.9). 
Comme $\delta \xi=-\tilde{\delta} C \xi$ est une divergence métrique, il résulte de (2.9), pour toute 1-forme $\xi$ à support compact, la formule importante [9]:

$$
\int_{W} \Lambda d \xi \cdot \eta=0
$$

\section{TRANSFORMATIONS INFINITÉSIMALES SYMPLECTIQUES ET CONFORMES SYMPLECTIQUES}

\section{Transformations infinitésimales symplectiques}

a) Etant donnée une variété symplectique $(W, F)$, étudions l'action d'une transformation infinitésimale (t.i.) $X$ sur la 2-forme fondamentale $F$. Si $\mathscr{L}(X)$ est l'opérateur de dérivation de Lie:

$$
\mathscr{L}(X) F=d i(X) F+i(X) d F,
$$

c'est-à-dire $F$ étant fermée

$$
\mathscr{L}(X) F=\operatorname{di}(X) F
$$

$X$ définit une t.i. symplectique (ou est un champ de vecteurs hamiltonien) si $F$ reste invariant par $X$. Pour qu'il en soit ainsi, il faut et il suffit la 1-forme $\mu(X)$ $=-i(X) F$ soit fermée.

Nous notons $L$ l'algèbre de Lie des transformations infinitésimales symplectiques de $(W, F), L_{0}$ celles des transformations infinitésimales symplectiques à supports compacts.

b) Soit $X, Y$ deux champs de vecteurs arbitraires de $W$. Evaluons l'image par $\mu$ de leur crochet, soit:

$$
\mu([X, Y])=-i([X, Y]) F=-\{\mathscr{L}(X) i(Y)-i(Y) \mathscr{L}(X)\} F .
$$

Or:

$$
\mathscr{L}(X) i(Y) F=\operatorname{di}(X) i(Y) F+i(X) d i(Y) F=\operatorname{di}(X) i(Y) F+i(X) \mathscr{L}(Y) F .
$$

Il vient ainsi:

$$
\mu([X, Y])=\operatorname{di}(X \wedge Y) F-i(X) \mathscr{L}(Y) F+i(Y) \mathscr{L}(X) F .
$$

Puisque pour des 2-formes $i\left(\mu^{-1}(\alpha)\right) \beta=i\left(\mu^{-1}(\beta)\right) \alpha$, on a :

$$
i(X \wedge Y) F=i\left(\mu^{-1}(F)\right) \mu(X \wedge Y)=\Lambda(\mu(X) \wedge \mu(Y)) .
$$

Ainsi pour tout couple de champs de vecteurs $X, Y$ de $W$ : 


$$
\mu([X, Y])=d \Lambda(\mu(X) \wedge \mu(Y))-i(X) \mathscr{L}(Y) F+i(Y) \mathscr{L}(X) F .
$$

Si $X, Y$ appartiennent à $L$, on a $\mathscr{L}(X) F=\mathscr{L}(Y) F=0$ et il vient :

$$
\mu([X, Y])=d \Lambda(\mu(X) \wedge \mu(Y)) .
$$

Soit $L^{*}$ le sous-espace de $L$ défini par les vecteurs dont l'image par $\mu$ est une 1 -forme exacte. Un élément de $L^{*}$ est encore dit un champ de vecteurs globalement hamiltonien. Il résulte de (3.3) que

$$
[L, L] \subset L^{*} .
$$

Si $X \in L$ et $Y \in L^{*}$, on a $\mu(Y)=d v$, où $v \in N$, et

$$
\Lambda(\mu(X) \wedge d v)=\mathscr{L}(X) v=-\Lambda d(v \mu(X)) .
$$

c) Désignons par $L_{0}^{*}$ le sous-espace de $L_{0}$ défini par les vecteurs $X$ tels que $\mu(X)=d u$ où $u \in N_{0}$. Il résulte de (3.3) que:

$$
\left[L, L_{0}\right] \subset L_{0}^{*} .
$$

Si $X \in L$ et $Y \in L_{0}^{*}\left(\operatorname{avec} \mu(Y)=d v, v \in N_{0}\right)$, la 1 -forme $v \mu(X)$ est à support compact et il résulte de (3.4) et (2.10) que:

$$
\int_{W} \Lambda(\mu(X) \wedge d v) \eta=0 .
$$

Ainsi $\Lambda(\mu(X) \wedge \mu(Y)) \in N_{1}$.

Soit $L_{1}$ le sous-espace de $L^{*}$ défini par les vecteurs $X$ tels que $\mu(X)=d u$ où $u \in N_{1}$. On a montré que:

$$
\left[L, L_{0}^{*}\right] \subset L_{1} .
$$

On établit de même:

$$
\left[L^{*}, L_{0}\right] \subset L_{1}
$$

Nous énonçons:

Proposition 1. $\left.1^{\circ}\right) \quad L^{*}$ est un idéal de $L$ tel que $L / L^{*}$ soit abélienne.

$\left.2^{\circ}\right)$ On $a$

$$
\left[L, L_{0}\right] \subset L_{0}^{*} .
$$

En particulier $L_{0}$ et $L_{0}^{*}$ sont des idéaux de $L$, et $L_{0} / L_{0}^{*}$ est abélienne.

$\left.3^{\circ}\right)$ On $a$ : 


$$
\text { (i) }\left[L, L_{0}^{*}\right] \subset L_{1}, \quad \text { (ii) }\left[L^{*}, L_{0}\right] \subset L_{1} \text {. }
$$

En particulier $L_{1}$ est un idéal de $L$.

d) Utilisons les théorèmes de G. de Rham. Soit $X$ un élément de $L,[\gamma]$ un élément de l'espace ${ }_{0} H_{1}(W ; R)$ d'homologie à support compact de $W$, admettant pour représentant un 1-cycle $\gamma$ différentiable compact. Nous posons

$$
J(X) \cdot[\gamma]=\int_{r} \mu(X) \in R .
$$

où le second membre ne dépend que de $X$ et $[\gamma]$. A tout élément $X$ de $L$ correspond ainsi un élément $J(X)$ de hom $\left({ }_{0} H_{1}(W ; R), R\right)$, c'est-à-dire de l'espace $H^{1}(W ; R)$ de cohomologie à supports fermés; $\mu(X)$ étant une 1 -forme fermée arbitraire, $J: X \rightarrow J(X)$ est une application linéaire de $L$ sur $H^{1}(W ; R)$ dont le noyau est $L^{*}$, d'après la définition de cet idéal. Ainsi l'espace $L / L^{*}$ est isomorphe à l'espace de cohomologie $H^{1}(W ; R)$ et

$$
\operatorname{dim} L / L^{*}=b_{1}(W),
$$

où $b_{1}(W)$ est le premier nombre de Betti de $W$ pour l'homologie à supports compacts.

Considérons l'espace $H_{1}(W ; R)$ d'homologie à supports fermés de $W$. A partir de hom $\left(H_{1}(W ; R), R\right)$, on définit de manière analogue une application linéaire $J_{0}$, de noyau $L_{0}^{*}$, de l'espace $L_{0}$ sur l'espace $H_{0}^{1}(W ; R)$ de cohomologie à supports compacts. On obtient ainsi [3]:

Proposition 2. $1^{\circ}$ ) L'espace $L / L^{*}$ est isomorphe à l'espace de cohomologie $H^{1}(W ; R)$ à supports fermés et

$$
\operatorname{dim} L / L^{*}=b_{1}(W) .
$$

$\left.2^{\circ}\right) \quad L^{\prime}$ espace $L_{0} / L_{0}^{*}$ est isomorphe á l'espace de cohomologie $H_{0}^{1}(W ; R) \grave{a}$ supports compacts et

$$
\operatorname{dim} L_{0} / L_{0}^{*}=b_{1}^{0}(W) .
$$

$b_{1}(W)$ (resp. $b_{1}^{0}(W)$ ) désigne ici le premier nombre de Betti de $W$ pour l'homologie à supports compacts (resp. fermés).

\section{Parenthèses de Poisson et produit scalaire invariant sur $L_{0}$}

a) Soit $N / R$ l'espace des classes de fonctions de $N$ modulo les constantes additives; nous notons $\pi: u \in N \rightarrow \pi(u)=\bar{u} \in N / R$ l'application canonique de $N$ sur $N / R$. Si $u \in N$, sa différentielle du ne dépend que de la classes $\bar{u}$ de $u$; nous la notons éventuellement $d \bar{u}$.

A chaque élément $X^{*}$ de $L$ correspond une 1 -forme exacte 


$$
\mu(X)=d u=d \bar{u}
$$

donc une classe $\bar{u}$ et inversement. Nour définissons ainsi un isomorphisme de l'espace vectoriel $L^{*}$ sur $N / R$. La structure d'algèbre de Lie induite sur $N / R$ est aisée à définir: si $\bar{u}, \bar{v} \in N / R$, il résulte de (3.3) que la fonction

$$
w=\Lambda(d \bar{u} \wedge d \bar{v})
$$

définit une classe $\bar{w}$ qui est, pour l'algèbre de Lie induite sur $N / R$, le crochet $[\bar{u}, \bar{v}]$.

A la fonction (4.1), on donne le nom de parenthèse de Poisson, par rapport à la structure symplectique, de $\bar{u}$ et $\bar{v}$, ou de deux représentants $u$ et $v$ dans $N$. On note cette parenthèse $\{\bar{u}, \bar{v}\}$ ou $\{u, v\}$. Ainsi:

$$
\{u, v\}=\{\bar{u}, \bar{v}\}=\Lambda(d u \wedge d v)=\Lambda(d \bar{u} \wedge d \bar{v}) .
$$

On a $[\bar{u}, \bar{v}]=\overline{\{\bar{u}, \bar{v}\}}$, de telle sorte que, pour ce crochet, $N / R$ est une algèbre de Lie isomorphe à $L^{*}$.

Soit $\left(x^{\alpha}, x^{\alpha}\right)$ une carte canonique de domaine $U$. On déduit immédiatement de (4.2) l'expression locale classique:

$$
\{u, v\}_{U}=\sum_{\alpha}\left(\partial_{\alpha} u \partial_{\bar{\alpha}} v-\partial_{\bar{\alpha}} u \partial_{\alpha} v\right) \quad\left(\partial_{i}=\partial / \partial x^{i}\right)
$$

b) La parenthèse de Poisson définit sur l'espace $N$ une structure d'algèbre de Lie et nous notons encore $N$ cette algèbre. Il suffit d'établir que l'identité de Jacobi est satisfaite. Si $u, v, w \in N$, il résulte en effet de l'identité de Jacobi vérifiée par $N / R$

$$
\{u,\{v, w\}\}+\{v,\{w, u\}\}+\{w,\{u, v\}\}=\text { const. }=C .
$$

Soit $U$ un domaine arbitraire de $W$; substituons à $u$ une fonction $u^{\prime}$ de $N$ telle que $\left.u\right|_{U}=\left.u^{\prime}\right|_{U}$ et nulle sur un autre ouvert de $W$. On a:

$$
\{u,\{v, w\}\}+\{v,\{w, u\}\}+\left.\{w,\{u, v\}\}\right|_{U}=0
$$

pour tout domaine $U$, ce qui établit l'identité de Jacobi.

\section{Comme}

$$
\{u, v\}=\Lambda d(u d v)
$$

on a, d'après (2.10),

$$
\left\{N, N_{0}\right\} \subset N_{1}
$$

Ainsi $N_{0}$ et $N_{1}$ sont des idéaux de $N ; N_{1}$ est le noyau de l'application linéaire de $N_{0}$ sur $R$ définie par: 


$$
u \in N_{0} \rightarrow \int_{W} u \eta \in R
$$

Par suite $\operatorname{dim} N_{0} / N_{1}=1$.

c) Si $W$ est non compacte, à chaque élément $X$ de $L_{0}^{*}$ correspond par $\mu(X)=d u$ une fonction unique $u$ de $N_{0}$. Il en résulte que $L_{0}^{*}$ est isomorphe à $N_{0}$ et $L_{1}$ à $N_{1}$.

Si $W$ est compacte $L_{0}=L, L_{0}^{*}=L^{*}$. Pour $X \in L^{*}, \mu(X)=d \bar{u}$ et la classe $\bar{u}$ contient un élément $u$ et un seul appartenant à $N_{1}$. Ainsi $L_{1}=L_{0}^{*}=L^{*}$ et $L^{*}$ est isomorphe à $N_{1}$.

Dans tous les cas, nous ferons correspondre à $X \in L_{0}^{*}$ une fonction unique $u$ à support compact pour $W$ non compacte, ou vérifiant (1.2) pour $W$ compacte. Nous poserons $u=\sigma(X)$, où $\sigma$ est un isomorphisme de $L_{0}^{*} \operatorname{sur} N_{0}$ dans le cas non compact, sur $N_{1}$ dans le cas compact.

d) Cela posé, soit $X, Y \in L_{0}^{*}$ auxquels correspondent les fonctions à supports compacts $u=\sigma(X), v=\sigma(Y)$. Nous introduisons sur $L_{0}^{*}$ la forme bilinéaire symétrique:

$$
\langle X, Y\rangle=\int_{W} u v \eta
$$

qui définit sur $L_{0}^{*}$ une structure d'espace préhilbertien.

Si $Z \in L$ et $\mu(Z)=\zeta$, il vient:

$$
\langle[Z, X], Y\rangle+\langle X,[Z, Y]\rangle=\int_{W} \Lambda((\zeta \wedge d u) v+(\zeta \wedge d v) u) \eta,
$$

soit

$$
\langle(Z, X], Y\rangle+\langle X,[Z, Y]\rangle=\int_{W} \Lambda(\zeta \wedge d(u v)) \eta=-\int_{W} \Lambda d(u v \zeta) \eta,
$$

où la 1-forme $u v \zeta$ est à support compact. Il résulte de (2.10):

$$
\langle[Z, X], Y\rangle+\langle X,[Z, Y]\rangle=0 .
$$

Ainsi le produit scalaire $\langle X, Y\rangle$ défini sur l'idéal $L_{0}^{*}$ de $L$ est invariant par l'action de $\operatorname{ad}(L)$.

L'existence de ce produit scalaire invariant va jouer un rôle important pour l'étude de la réductivité de certaines sous-algèbres de $L_{0}^{*}$

\section{Transformations infinitésimales conformes symplectiques}

a) $X$ définit une t.i. conforme symplectique s'il existe $a \in N$ telle que

$$
\mathscr{L}(X) F+a F=0,
$$


soit

$$
d i(X) F+a F=0
$$

Par différentiation extérieure, il vient:

$$
d a \wedge F=0 .
$$

$F$ étant partout de rang $2 n$, il résulte d'un théorème classique de Lepage que, pour $n>1,(5.1)$ entraîne $d a=0$, c'est-à-dire $a=$ const. $=K$.

Pour tout $n$, nous notons $L^{c}$ et appelons_-par abus de langage_-algèbre de Lie des t.i. conformes symplectiques, l'algèbre de Lie des champs de vecteurs $X$ tels que:

$$
\mathscr{L}(X) F+K_{X} F=0 \quad\left(K_{X}=\text { const. }\right) .
$$

Pour $X \in L^{c}$ et $Y \in L$, la relation (3.2) s'écrit :

$$
\mu([X, Y])=d \Lambda(\mu(X) \wedge \mu(Y))+K_{X} \mu(Y) .
$$

$\mu(Y)$ étant fermée, $\mu([X, Y])$ est fermée et $[X, Y] \in L$. Ainsi $L$ est un idéal de $L^{c}$. Si $Y \in L^{*}$, le second membre de (5.3) est une 1 -forme exacte et $[X, Y]$ $\in L^{*} ; L^{*}$ est aussi un idéal de $L^{c}$. La même conclusion s'étend, à partir de (5.3), à $L_{0}, L_{0}^{*}, L_{1}$.

Proposition 1. Si $L^{c}$ est l'algèbre de Lie des transformations infinitésimales conformes symplectiques de $W$ les algèbres $L, L^{*}, L_{0}, L_{0}^{*}, L_{1}$ sont des idéaux de $L^{c}$.

b) Si $X \in L^{c},(5.2)$ peut s'écrire:

$$
K_{X} F=d \mu(X) .
$$

Si $F$ n'est pas exacte (en particulier si $W$ est compacte), il résulte de (5.4) que $K_{X}=0$ pour tout élément $X$ de $L^{c}$. Ainsi dans ce cas $L^{c}$ coïncide avec $L$.

Examinons le cas où $F$ est exacte. On a $F=d \alpha$, où $\alpha$ est une 1 -forme de $W$; si $X_{0}=\mu^{-1}(\alpha)$, il vient

$$
F=d \mu\left(X_{0}\right)
$$

et $X_{0}$ appartient à $L^{c}$ avec $K_{X_{0}}=1$. Si $X \in L^{c}$, on a :

$$
K_{X} F=d \mu(X)=d \mu\left(K_{X} X_{0}\right),
$$

et par suite:

$$
d \mu\left(X-K_{X} X_{0}\right)=0 .
$$

Ainsi $X-K_{X} X_{0} \in L$. Tout élément $X$ de $L^{c}$ admet une décomposition unique 
en somme d'un élément proportionnel à $X_{0}$ et d'un élément de L:

$$
X=K_{X} X_{0}+Y \quad(Y \in L) .
$$

Si $X^{\prime}$ est un autre élément de $L^{c}$ :

$$
X^{\prime}=K_{X^{\prime}} X_{0}+Y^{\prime} \quad\left(Y^{\prime} \in L\right) .
$$

Il vient:

$$
\left[X, X^{\prime}\right]=K_{X}\left[X_{0}, Y^{\prime}\right]+K_{X^{\prime}}\left[Y, X_{0}\right]+\left[Y, Y^{\prime}\right]
$$

où chaque terme du second membre appartient à $L$. Par suite:

$$
\left[L^{c}, L^{c}\right] \subset L .
$$

Inversement, soit $Y$ un élément arbitraire de $L$; on déduit de (5.3) pour $X_{0}$ et $Y$ :

$$
\mu(Y)=\mu\left(\left[X_{0}, Y\right]\right)-d \Lambda\left(\mu\left(X_{0}\right) \wedge \mu(Y)\right),
$$

soit:

$$
Y=\left[X_{0}, Y\right]-\mu^{-1}\left\{d \Lambda\left(\mu\left(X_{0}\right) \wedge \mu(Y)\right\}\right.
$$

On sait [3]—et nous le réétablirons ultérieurement—que $L^{*}$ est l'idéal dérivé $[L, L]$ de $L$. Le premier terme du second membre de (5.7) appartient à $\left[L^{c}, L^{c}\right]$, le second à $L^{*}$, donc à $[L, L]$. Il en résulte :

$$
L \subset\left[L^{c}, L^{c}\right] \text {. }
$$

Ainsi $\left[L^{c}, L^{c}\right]=L$.

Proposition 2. Si la 2-forme fondamentale $F$ d'une variété symplectique est exacte, $L^{c}$ est, en tant qu'espace vectoriel, somme directe de $L$ et d'un sousespace $C_{0}$ de dimension 1 :

$$
L^{c}=L \oplus C_{0}
$$

En particulier $\operatorname{dim} . L^{c} / L=1$. On a de plus:

$$
\left[L^{c}, L^{c}\right]=L .
$$




\section{REDUCTIVITE D'ALGÈBRE DE TRANSFORMATIONS INFINITÉSIMALES SYMPLECTIQUES}

\section{Semi-simplicité et réductivité}

Les algèbres de Lie $A$ envisagées dans ce paragraphe et le suivant sont des algèbres de Lie quelconques sur le corps des réels, de dimension finie ou infinie. A l'algèbre $A$, nous associons la suite des dérivées:

$$
A^{(0)}=A, \quad A^{(1)}=[A, A], \cdots, \quad A^{(p)}=\left[A^{(p-1)}, A^{(p-1)}\right], \cdots .
$$

a) Soit $I$ un idéal de $A$. Il résulte immédiatement de l'identité de Jacobi que $I^{(1)}$ est aussi un idéal de $A$; de plus, si $I^{(p-1)}$ est un idéal de $A,\left(I^{(p-1)}\right)^{(1)}$ $=I^{(p)}$ est un idéal de $A$. On voit ainsi par récurrence que pour tout $p, I^{(p)}$ est un idéal de $A$.

L'idéal $I$ est abélien si $I^{(1)}=0$; il est résoluble s'il existe un entier positif $p$ tel que $I^{(p)}=0$.

b) Un idéal $P$ de $A$ est dit primitif si, pour tout idéal $I$ de $A$ tel que $I^{(1)}$ $\subset P$, on a nécessairement $I \subset P$.

Il résulte de cette définition que $A$ est un idéal primitif trivial et que toute intersection d'idéaux primitifs de $A$ est un idéal primitif de $A$.

$P$ étant un idéal primitif, soit $I$ un idéal de $A$ tel que $I^{(p)} \subset P ; I^{(p-1)}$ est alors un idéal de $A$ tel que $\left(I^{(p-1)}\right)^{(1)} \subset P$, donc $I^{(p-1)} \subset P$. On voit ainsi de proche en proche que $I \subset P$. En particulier, tout idéal résoluble de $A$ est contenu dans $P$.

Nous sommes conduits à la définition suivante

Définition. On appelle radical de $A$ l'intersection $R_{A}$ de tous les idéaux primitifs de $A$.

Si $A$ est de dimension finie, $R_{A}$ coïncide bien avec le radical usuel $R$ de $A$, défini comme le plus grand idéal résoluble de $A$. En effet soit $I$ un idéal de $A$ tel que $I^{(1)} \subset R ; I$ est résoluble donc contenu dans $R$ et $R$ est primitif; ainsi $R_{A} \subset R$. Inversement $R$ résoluble est contenu dans tout idéal primitif de $A$, donc dans leur intersection $R_{A}$ et $R \subset R_{A}$.

c) Définition. Une algèbre de Lie $A$ est dite semi-simple si son radical $R_{A}$ est nul ou-ce qui est équivalent—si elle n'admet pas d'idéal abélien $\neq\{0\}$.

Montrons l'équivalence de ces deux conditions. Supposon $R_{A}=0$ et soit $I$ un idéal abélien de $A ; I$ étant résoluble est contenu dans tout idéal primitif, donc dans $R_{A}$ et $I=\{0\}$.

Inversement supposons que $\{0\}$ soit le seul idéal abélien de $A$; alors $\{0\}$ est, par définition, un idéal primitif de $A$ et c'est le plus petit idéal primitif, donc $R_{A}=0$.

La réductivité d'une algèbre de Lie $A$ peut être définie de la manière suivante: 
Définition. Une algèbre de Lie $A$ est dite réductive si elle est somme directe de son centre et d'une algèbre semi-simple.

On a

$$
A=C \oplus B,
$$

où $C$ est le centre de $A$ et $B$ une algèbre semi-simple. En fait $B$ est nécessairement un idéal de $A$, car $[A, B]=[B, B] \subset B$.

On a

Proposition. Pour qu'une algèbre de Lie A soit réductive, il faut et il suffit qu'elle soit somme directe d'un idéal abélien et d'un idéal semi-simple.

Montrons que la condition est suffisante. On a:

$$
A=\Gamma \oplus B,
$$

où $\Gamma$ est un idéal abélien et $B$ un idéal semi-simple; $\Gamma$ est manifestement contenu dans le centre $C$ de $A$. Décomposons $C$ selon (6.2); il vient

$$
C=\Gamma \oplus M \quad(M \subset B) .
$$

On a $[M, B]=0$ et $M$ appartient au centre de l'algèbre semi-simple $B$. Ainsi $M=0$ et $\Gamma=C$.

\section{Algèbres de Lie admettant un produit scalaire invariant}

Nous considérons dans ce paragraphe des algèbres de Lie $A$ admettant un produit scalaire $\langle X, Y\rangle$ invariant par ad $(A)$ et définissant sur $A$ une structure d'espace préhilbertien. L'hypothèse d'invariance se traduit par la relation

$$
\langle[Z, X], Y\rangle+\langle X,[Z, Y]\rangle=0
$$

pour tous $X, Y, Z \in A$.

a) Nous établissons d'abord la proposition suivante:

Proposition. Soit $A$ une algèbre de Lie admettant un produit scalaire invariant.

$\left.1^{\circ}\right)$ Si I est un idéal de $A$, son centre est contenu dans le centre $C$ de $A$.

$2^{\circ}$ ) En particulier tout idéal abélien de $A$ est contenu dans $C$. Pour que $A$ soit semi-simple, il faut et il suffit que $C=0$.

Soit $I$ un idéal de $A, \Gamma$ son centre. Si $X \in A, Y \in \Gamma, c \in \Gamma$ on a d'après (7.1):

$$
\langle[c, X],[c, X]\rangle+\langle X,[c,[c, X]]\rangle=0 .
$$

Or $[c, X] \in I$ et par la suite $[c,[c, X]]=0$. Il en résulte $[c, X]=0$ pour tout $X \in A$. Ainsi $\Gamma \subset C$, ce qui démontre la proposition.

b) On en déduit 
Théorème 1. L'algèbre dérivée $A^{(1)}$ d'une algèbre $A$ admettant un produit scalaire invariant est semi-simple.

En effet soit $\Gamma$ le centre de $A^{(1)}$; d'après la proposition précédente, il est contenu dans le centre de $A$ et si $X \in A, c \in \Gamma$, on a $[c, X]=0$. Si $X, X^{\prime} \in A$, il résulte de (7.1):

$$
\left\langle\left[X, X^{\prime}\right], c\right\rangle+\left\langle X^{\prime},[X, c]\right\rangle=0,
$$

donc

$$
\left[\left[X, X^{\prime}\right], c\right]=0 .
$$

$c$ est orthogonal à $A^{(1)}$ donc à lui-même et $c=0$. Ainsi $\Gamma=0$. Le produit scalaire de $A$ induisant sur $A^{(1)}$ un produit scalaire invariant, $A^{(1)}$ est semisimple d'après le $2^{\circ}$ de la proposition précédente.

c) On a:

Théorème 2. Toute algèbre de Lie A admettant un produit scalaire invariant et nilpotente (resp. résoluble) est abélienne.

En effet, si $A$ est nilpotente, soit $A_{(p)}$ le premier terme nul de la série centrale descendante. Si $p>1$, on a pour $X \in A, Y \in A, Z \in A_{(p-1)}$ :

$$
\langle[X, Y], Z\rangle+\langle Y,[X, Z]\rangle=0,
$$

donc:

$$
\langle[X, Y], Z\rangle=0 .
$$

Ainsi $A_{(p-1)}$ est orthogonal à $A_{(1)}$ donc à lui-même et $A_{(p-1)}=0$, contrairement à l'hypothèse faite sur $p$. Ainsi $A_{(1)}=0$ et $A$ est abélienne.

Si $A$ est résoluble, soit $A^{(p)}$ le premier terme nul de la série dérivée. Si $p>1, A^{(p-1)}=\left[A^{(p-2)}, A^{(p-2)}\right]$ est semi-simple et abélienne, donc nulle. C'est que $A^{(1)}=0$ et $A$ est abélienne.

d) Théorème 3. Soit $A$ une algèbre de Lie admettant un produit scalaire invariant.

$\left.1^{\circ}\right)$ Si $C$ est le centre de $A, A / C$ est semi-simple. Si $A$ est somme directe de $C$ et d'un idéal, $A$ est réductive.

$2^{\circ}$ ) Si $C$ est de dimension finie, $A$ est réductive.

$\left.3^{\circ}\right)$ Si $A^{(1)}$ est de dimension finie, $A$ est réductive.

En effet soit $J$ un idéal abélien de $A / C, I$ l'ensemble des représentants dans $A$ des éléments de $J ; I$ est un idéal de $A$ tel que $[I, I] \subset C \cap A^{(1)}$. Or $A^{(1)}$ étant semi-simple, $C \cap A^{(1)}=\{0\}$. Ainsi $I$ est abélien, donc central et $J=\{0\}$.

S'il existe un idéal $B$ de $A$ tel que $A=C \oplus B, B$ est isomorphe à $A / C$ donc semi-simple et $A$ est réductive.

Supposons $C$ de dimension finie; c'est un sous-espace complet de l'espace 
préhilbertien $A$. Il existe un orthocomplément $B$ de $C$ dans $A ; C$ étant invariant par $\operatorname{ad}(A), B$ l'est aussi, donc est un idéal de $A$ et $A$ est réductive.

Supposons maintenant $A^{(1)}$ de dimension finie; il existe un orthocomplément $\Gamma \operatorname{de} A^{(1)}$ dans $A$ et

$$
A=\Gamma \oplus A^{(1)},
$$

où $\Gamma$ est un idéal de $A$. On a $[\Gamma, \Gamma] \subset \Gamma \cap A^{(1)}=\{0\}$ donc $\Gamma$ est abélien. Ainsi $A$, somme directe d'un idéal abélien $\Gamma$ et d'un idéal semi-simple $A^{(1)}$, est réductive.

\section{8. $\quad L_{0}^{*}$ et ses sous-algébres}

a) Nous avons vu $(\S 4)$ que $L_{0}^{*}$ admet un produit scalaire invariant par $\operatorname{ad}(L)$ définissant $L_{0}^{*}$ comme espace préhilbertien. Par suite, toute sous-algèbre $A$ de $L_{0}^{*}$ admet un produit scalaire invariant par $\operatorname{ad}(A)$ qui est la restriction à $A \mathrm{du}$ produit scalaire précédent, et elle satisfait aux hypothèses du $\S 7$. Il en résulte en particulier:

Théorème. Soit $A$ une sous-algèbre de $L_{0}^{*}$ de centre $C$.

$\left.1^{\circ}\right)$ L'algèbre dérivée $A^{(1)}$ de $A$ est semi-simple; $A / C$ est semi-simple.

$2^{\circ}$ ) Si le centre $C$ de $A$ est de dimension finie ou si $A^{(1)}$ est de dimension finie, l'algèbre $A$ est réductive.

On voit que si $W$ est non compacte (resp. compacte), toute sous-algèbre $A$ de $N_{0}$ (resp. $N_{1}$ ) dont le centre est de dimension finie est réductive. Il en est bien entendu ainsi pour les algèbres de dimension finie.

b) Considérons l'action sur $L_{0}^{*}$ d'un élément $Z$ de $L$. Si $X \in L_{0}^{*}$, on a $\mu(X)$ $=d u$, où $u \in N_{0}$, et

$$
\operatorname{ad}(Z) X=\mu^{-1}\left(d \Lambda(\mu(Z) \wedge d u)=\mu^{-1}(d \mathscr{L}(Z) u),\right.
$$

où $\mathscr{L}(Z) u$ vérifie :

$$
\int_{W} \mathscr{L}(Z) u \eta=0
$$

Pour que $\operatorname{ad}(Z) X=0$, il faut et il suffit que $\mathscr{L}(Z) u=$ const. $=C$; mais d'après (8.2), $C=0$ donc

$$
\mathscr{L}(Z) u=0
$$

Supposons que $\operatorname{ad}(Z) X=0$ pour tout $X \in L_{0}^{*}$; on a (8.3) pour tout $u \in N_{0}$, soit

$$
i\left(\mu^{-1}(d u)\right) \mu(Z)=0 .
$$

Soit $x$ un point arbitraire de $W$; on peut trouver $u \in N_{0}$ tel que $\mu^{-1}(d u)$ soit un 
vecteur arbitraire donné en $x$. Il en résulte $\mu(Z)_{x}=0$. Ainsi $Z=0$ et le centralisateur de $L_{0}^{*}$ dans $L$ est nul.

$L_{0}^{*}$ satisfaisant à l'hypothèse de la proposition du $\S 7$ et étant de centre nul, il vient

Proposition. Le centralisateur de $L_{0}^{*}$ dans $L$ est nul. En particulier $L_{0}^{*}$ est semi-simple.

c) Soit $N$ un entier $\geq 1$. On déduit de (8.1) par itération:

$$
\operatorname{ad}(Z)^{N} X=\mu^{-1}\left(d \mathscr{L}(Z)^{N} u\right),
$$

et on voit comme au $b$ que, pour que $\operatorname{ad}(Z)^{N} X=0$, il faut et il suffit que:

$$
\mathscr{L}(Z)^{N} u=0 .
$$

Nous notons que si $u, v \in N_{0}$, on a la formule de dérivation:

$$
\mathscr{L}(Z)(u v)=\mathscr{L}(Z) u \cdot v+u \mathscr{L}(Z) v .
$$

Nous allons établir [1]

Théorème. Il n'existe aucun élément de L non nul qui soit nilpotent dans son action sur $L_{0}^{*}$.

Si $Z$ est un élément de $L$ nilpotent dans son action sur $L_{0}^{*}$, soit $N \geq 1$ l'entier minimum tel que pour tout $X \in L_{0}^{*}$, on ait $\operatorname{ad}(Z)^{N} X=0$. Il est équivalent de dire que pour tout $u \in N_{0}$

$$
\mathscr{L}(Z)^{N} u=0
$$

Si $N=2$, on a pour tout $u \in N_{0}$ :

$$
\mathscr{L}(Z)^{2} u^{2}=2 u \mathscr{L}(Z)^{2} u+2(\mathscr{L}(Z) u)^{2} .
$$

On en déduit que $\mathscr{L}(Z) u=0$ pour tout $u \in N_{0}$, ce qui contredit l'hypothèse faite sur N. Si $N>2$, on a pour tout $u, v \in N_{0}$

$$
\mathscr{L}(Z)^{N}(u v)=\mathscr{L}(Z)^{N} u \cdot v+\sum_{a=1}^{N-1} C_{N}^{a} \mathscr{L}(Z)^{N-a} u \cdot \mathscr{L}(Z)^{a} v+u \mathscr{L}(Z)^{N} v .
$$

On en déduit:

$$
\sum_{a=1}^{N-1} C_{N}^{a} \mathscr{L}(Z)^{N-a} u \cdot \mathscr{L}(Z)^{a} v=0
$$

Prenons $v=\mathscr{L}(Z)^{N-2} u$. Il vient:

$$
\sum_{a=1}^{N-1} C_{N}^{a} \mathscr{L}(Z)^{N-a} u \mathscr{L}(Z)^{N-2+a} u=0
$$

Pour $a=2, \cdots, N$ les termes de cette somme sont nuls. Il reste le terme cor- 
respondant à $a=1$, soit

$$
N\left(\mathscr{L}(Z)^{N-1} u\right)^{2}=0
$$

Ainsi $\mathscr{L}(Z)^{N-1} u=0$, ce qui contredit l'hypothèse faite sur $N$.

On a donc toujours $N=1$ et $Z$ appartient au centralisateur de $L_{0}^{*}$ dans $L$, ce qui démontre le théorème d'après la proposition précédente.

\section{Sous-algèbres propres de $N$}

a) Une fonction $u \in N$ sera dite propre si $u^{-1}(c)$ est compact pour tout réel $c \neq 0$. Une sous-algèbre de Lie $\mathscr{A}$ de $N$ est propre si see éléments sont des fonctions propres. Toute sous-algèbre de $N_{0}$ est manifestement propre.

Nous allons établir le lemme suivant

Lemme. Si $u, v \in N$ et si $u$ est propre, alors $\{u, v\}=a^{2} \geq 0$ entraîne $\{u, v\}=0$.

Soit $x_{0}$ un point de $W$ tel que $u\left(x_{0}\right) \neq 0$. Considérons la sous-variété compacte $K$ définie par les points $x$ de $W$ tels que $u(x)=u\left(x_{0}\right)$. Le vecteur $X=\mu^{-1}(d u)$ vérifie $\mathscr{L}(X) u=0$. Si pour $x \in K, X_{x}$ est $\neq 0, x$ est un point régulierde $K$ et $X_{x}$ est tangent à $K$; si $X_{x}=0, x$ est singulier pour $K$. Nous notons $\stackrel{K}{K}$ l'ouvert de $K$ défini par ses points réguliers; l'ensemble des points singuliers de $K$ est compact et en ces points $\{u, v\}=0$.

Soit $k$ la densité scalaire associée à $\eta, \tau$ l'élément de volume induit sur $K$ par $\eta$. Au voisinage de $x \in \stackrel{\circ}{K}$, on a en coordonnées locales, d'après (2.9):

$$
\{u, v\}=\frac{1}{k} \partial_{i}\left(v X^{i} k\right)
$$

$X$ étant tangent à $\stackrel{\circ}{K}$, on en déduit que si $\left\{x^{a}\right\}(a=1, \cdots, 2 n-1)$ est un système de coordonnées locales de $\stackrel{K}{K}$ dont le domaine contient $x$, on a sur ce domaine

$$
\{u, v\} k=\partial_{a}\left(v X^{a} k\right)
$$

On peut construire une suite $M_{1} \subset M_{2} \subset \ldots \subset M_{r} \subset \ldots$ de sous-variétés à bord portées par $\stackrel{\circ}{K}$, telles que $\lim M_{r}=K$ et que aire $\left(\partial M_{r}\right)$ (au sens de l'élement induit par $\tau$ ) soit bornée par un nombre fixe $A$. On déduit de (9.1):

$$
\int_{M_{r}} a^{2} \tau=\int_{M_{r}}\{u, v\} \tau=\operatorname{flux}_{\partial M_{r}}(v X)
$$

Etant donné $\varepsilon>0$. on peut choisir $r$ suffisamment grand pour que flux $\partial_{\partial M_{r}}$ $(v X)<\varepsilon$; on en déduit, d'après (9.2), que pour chaque indice $r$, on a : 


$$
\int_{M_{r}} a^{2} \tau<\varepsilon
$$

Ainsi:

$$
\int_{M_{r}} a^{2} \tau=0
$$

et $a^{2}=0$ sur tout $M_{r}$, donc sur $K$.

En tous les points $x$ de l'ouvert de $W$ défini par $u(x) \neq 0$, on a $\{u, v\}=0$. Il en résulte que $\{u, v\}=0$ sur $S(u)$. Sur le complémentaire $\lceil S(u)$, on a aussi manifestement $\{u, v\}=0$ d'après la définition même de la parenthèse de Poisson. Ainsi $\{u, v\}=0$ sur $W$.

b) De ce lemme, on peut déduire la proposition suivante semblable à celle du $\S 7$ :

Proposition. Soit $\mathscr{A}$ une sous-algèbre de Lie propre de $N$.

$\left.1^{\circ}\right)$ Si I est un idéal de $\mathscr{A}$, son centre est contenu dans le centre $\mathscr{C}$ de $\mathscr{A}$.

$2^{\circ}$ ) En particulier tout idéal abélien de $\mathscr{A}$ est contenu dans $\mathscr{C}$. Pour que $\mathscr{A}$ soit semi-simple il faut et il suffit que $\mathscr{C}=0$.

Soit $I$ un idéal de $\mathscr{A}, \Gamma$ son centre. Si $u \in \mathscr{A}, c \in \Gamma$, on a $\{c, u\} \in I$, et

$$
\{c,\{c, u\}\}=0 \text {. }
$$

On en déduit:

$$
\{c,\{c, u\} u\}=(\{c, u\})^{2} .
$$

$c$ étant propre, on déduit du lemme précédent que $\{c, u\}=0$ pour tout $\mathrm{u} \in \mathscr{A}$. Ainsi $\Gamma \subset \mathscr{C}$, ce qui démontre la proposition

Il en résulte

Théorème 1. L'algèbre dérivée $\mathscr{A}^{(1)}$ d'une sous-algèbre propre $\mathscr{A}$ de $N$ est semi-simple.

En effet soit $\Gamma$ le centre de $\mathscr{A}^{(1)}$; d'après la proposition précédente, il est contenu dans le centre de $\mathscr{A}$. Si $c \in \Gamma$, il existe $u, v \in \mathscr{A}$ tels que $c=\{u, v\}$ et l'on a:

$$
\{u,\{u, v\}\}=0
$$

On en déduit:

$$
\{u,\{u, v\} v\}=(\{u, v\})^{2}
$$

$u$ étant propre, on a $\{u, v\}=c=0$ d'après le lemme et $\Gamma=0 ; \mathscr{A}^{(1)}$ est semisimple d'après le $2^{\circ}$ de la proposition précédente.

Théorème 2. Toute sous-algèbre de Lie propre $\mathscr{A}$ de $N$ qui est nilpotente (resp. résoluble) est abélienne. 
En effet, si $\mathscr{A}$ est nilpotente, soit $\mathscr{A}_{(p)}$ le premier terme nul de la série centrale descendante. Si $p>1$, l'algèbre $\mathscr{A}_{(p-1)}$ est engendrée par les $\{u, v\}$, où $u \in \mathscr{A}$ et $v \in \mathscr{A}_{(p-2)}$. Comme $\mathscr{A}_{(p)}=0$, on a

$$
\{u,\{u, v\}\}=0
$$

et par suite:

$$
\{u,\{u, v\} v\}=(\{u, v\})^{2} .
$$

$u$ étant propre, on a $\{u, v\}=0$ pour tout $u \in \mathscr{A}, v \in \mathscr{A}_{(p-2)}$, donc $\mathscr{A}_{(p-1)}=0$ ce qui contredit l'hypothèse faite sur $p$. Ainsi $\mathscr{A}_{(1)}=0$ et $\mathscr{A}$ est abélienne.

Si $\mathscr{A}$ est résoluble, soit $\mathscr{A}^{(p)}$ le premier terme nul de la série dérivée. Si $p$ $>1, \mathscr{A}^{(p-1)}=\left[\mathscr{A}^{(p-2)}, \mathscr{A}^{(p-2)}\right]$ est semi-simple et abélienne donc nulle. C'est que $\mathscr{A}^{(1)}=0$ et $\mathscr{A}$ est abélienne.

On notera que les résultats précédents s'appliquent aux sous-algèbres $\mathscr{A}$ de $N_{0}$ et par suite aux sous-algèbres $A$ de $L_{0}^{*}$. On retrouve ainsi pour ces algèbres, par une voie différente, les résultats provenant des théorèmes 1 et 2 du $\S 7$.

On déduit immédiatement de la proposition précédente et du théorème 1.

Théorème 3. Soit $\mathscr{A}$ une sous-algèbre de Lie propre de $N$.

$\left.1^{\circ}\right)$ Si $\mathscr{C}$ est le centre de $\mathscr{A}$, l'algèbre de Lie $\mathscr{A} / \mathscr{C}$ est semi-simple.

$\left.2^{\circ}\right)$ Si $\mathscr{A}$ est de dimension finie, elle est réductive.

Le $1^{\circ}$ ) résulte du raisonnement même qui conduit au $1^{\circ}$ ) du théorème $3 \mathrm{du}$ $\S 7$. Le $2^{\circ}$ ) est une conséquence classique du $1^{\circ}$ ) pour une algèbre de Lie de dimension finie.

On retrouve ainsi par une autre voie que toute sous-algèbre de dimension finie de $L_{0}^{*}$ est réductive.

\section{LES IDÉAUX DE $L$}

\section{Introduction des $(2 n-1)$-formes}

a) Supposons $W$ non compacte et considérons un élément $u$ de $N$. La variété $W$ étant connexe, non compacte, son $2 n^{e}$ groupe de cohomologie réelle à supports non restreints est trivial. Il existe par suite sur $W$ une $(2 n-1)$ forme $\psi_{u}$ telle que $u \eta=d \psi_{u}$.

$W$ étant compacte ou non, considérons un élément $u$ de $N_{1}$. D'après (1.2), il existe sur $W$ une $(2 n-1)$-forme $\psi_{u}$, à support $S\left(\psi_{u}\right)$ compact, telle que $u \eta=d \psi_{u}$.

Ainsi si $W$ est non compacte (resp. quelconque) étant donné un élément $X$ de $L^{*}$ (resp. $\left.L_{1}\right)$, il existe une $(2 n-1)$-forme $\psi$ de $W$ (resp. à support compact) telle que, d'après (1.7): 


$$
\mu(X)=d u,
$$

avec:

$$
u=* d \mu=-\delta * \psi .
$$

En ce qui concerne les supports, on a

$$
S(X) \subset S(u) \subset S(\psi) .
$$

b) Soit $X=\mu^{-1}(d u) \in L^{*}\left(\right.$ resp. $\left.L_{1}\right)$ et $Y \in L$; on a $[X, Y] \in L^{*}$ (resp. $\left.L_{1}\right)$ et :

$$
\mu([X, Y])=d w,
$$

où d'après (2.9):

$$
w=\Lambda(d u \wedge \mu(Y))=\Lambda d(u \mu(Y))=\delta(u \mu(Y)) .
$$

Une $(2 n-1)$-forme de $W$ (resp. à support compact) correspondant à $w$ est:

$$
\psi_{w}=*(u \mu(Y)) .
$$

En particulier:

$$
S\left(\psi_{w}\right)=S(u) \cap S(Y)
$$

\section{Lemme de Calabi généralisé}

Nous nous proposons d'établir un lemme important qui nous sera utile à différentes reprises et qui constitue une généralisation d'un résultat de Calabi.

a) Soit $U^{\prime}$ un domaine contractile de coordonnées locales arbitraires $\left\{x^{i}\right\}$, où $x^{i} \in N$. Soit $U$ un autre domaine contractile tel que $\bar{U}^{\prime} \subset U$. Introduisons une fonction $a$ à valeurs $\geq 0$, égale à 1 sur $\bar{U}^{\prime}$ et à support compact $S(a)=$ $K \subset U$. Les fonctions $\bar{w}^{(i)}=a x^{i}$ appartiennent à $N_{0}$, sont à supports dans $K$ et telles que $\left.\bar{w}^{(i)}\right|_{U^{\prime}}=\left.x^{i}\right|_{U^{\prime}}$. Considérons une fonction $b$ à valeurs $\geq 0$, non identiquement nulle, à support compact $S(b) \subset U-K$; il existe des constantes $d_{i}$ telles que:

$$
\int_{W}\left(\bar{w}^{(i)}-d_{i} b\right) \eta=0 .
$$

Les fonctions $w^{(i)}=\bar{w}^{(i)}-d_{i} b$ appartiennent à $N_{1}$, sont à supports $S\left(w^{(i)}\right) \subset$ $U$ et telles que $\left.w^{(i)}\right|_{U^{\prime}}=\left.x^{i}\right|_{U^{\prime}}$.

b) Lemme. Soit $U, U^{\prime}$ deux domaines contractiles de $W$, avec $\bar{U}^{\prime} \subset U$. Donnons-nous 2 fonctions $w^{(i)} \in N_{1}$, à supports $S\left(w^{(i)}\right) \subset U$, telles que les $x^{i}=$ $\left.w^{(i)}\right|_{U^{\prime}}$ définissent une carte locale de domaine $U^{\prime}$. Si u est un élément de $N_{1}$ 
tel que $S(u) \subset U^{\prime}$, il existe $2 n$ fonctions $v_{(i)} \in N_{1}$, à supports $S\left(v_{(i)}\right) \subset U$ telles que l'on ait:

$$
u=\sum_{i}\left\{v_{(i)}, w^{(i)}\right\}
$$

Comme $u$ vérifie (1.2) et est à support $S(u) \subset U^{\prime}$, il existe une $(2 n-1)$ forme $\psi$ à support compact $S(\psi)$ contenu dans $U^{\prime}$ telle que:

$$
u \eta=d \psi
$$

Nous posons $* \psi=-\xi$ de telle sorte que $u=\delta \xi$.

Introduisons un instant une structure presque kählerienne subordonnée à la structure symplectique. Avec les notations du $\S 2$, il vient en coordonnées locales sur $U^{\prime}$ :

$$
u=\nabla_{j}\left(F^{j i} \xi_{i}\right)
$$

D'autre part, pour des fonctions $\bar{v}_{(i)}$ à supports $S\left(\bar{v}_{(i)}\right) \subset U^{\prime}$, on a $S\left(\left\{\bar{v}_{(i)}, w^{(i)}\right\}\right)$ $\subset U^{\prime}$ et d'après l'expression des parenthèses de Poisson:

$$
\sum_{i}\left\{\bar{v}_{(i)}, w^{(i)}\right\}=\sum_{i} F^{j k} \partial_{j} \bar{v}_{(i)} \partial_{k} w^{(i)}
$$

Or, pour les coordonnées locales $\left\{x^{i}\right\}$ choisies sur $U^{\prime}$, on a $\partial_{k} w^{(i)}=\delta_{k}^{i}$ sur $U^{\prime}$; $F$ étant cofermée métriquement, il en résulte:

$$
\sum_{i}\left\{\bar{v}_{(i)}, w^{(i)}\right\}=\sum_{i} F^{j i} \nabla_{j} \bar{v}_{(i)}=\nabla_{j}\left(\sum_{i} F^{j i} \bar{v}_{(i)}\right) .
$$

En posant $\bar{v}_{(i)}=\xi_{i}$ et compte-tenu de $\Gamma_{j k}^{i} F^{j k}=0$, on a d'après (11.2)

$$
u=\sum_{i}\left\{\bar{v}_{(i)}, w^{i}\right\}
$$

où $\bar{v}_{(i)}$ est à support compact $S\left(\bar{v}_{(i)}\right) \subset U^{\prime}$.

Désignons par $K \subset U$ un compact tel que $S\left(w^{(i)}\right) \subset K$. Considérons une fonction $h$ à valeurs $\geq 0$, non identiquement nulle, à support compact $S(h) \subset$ $U-K$; il existe des constantes $c_{i}$ telles que:

$$
\int_{W}\left(\bar{v}_{(i)}-c_{i} h\right) \eta=0 \text {. }
$$

Pour $v_{(i)}=\bar{v}_{(i)}-c_{i} h$, la considération des supports donne $\left\{v_{(i)}, w^{(i)}\right\}=$ $\left\{\bar{v}_{(i)}, w^{(i)}\right\}$. Ainsi

$$
u=\sum_{i}\left\{v_{(i)}, w^{(i)}\right\}
$$

où $v_{(i)} \in N_{1}$ est à support $S\left(v_{(i)}\right) \subset U$, ce qui démontre le lemme. 


\section{Idéaux dérivées}

Calabi [3] a étudié les idéaux dérivés de $L, L^{*}, L_{0}, L_{0}^{*}, L_{1}$. Pour être complet, nous rappelons ici cette étude. Nous supposons $W$ non compacte, mais les énoncés sont aussi valables daus le cas compact.

a) Introduisons un recouvrement $\left\{U_{\nu}\right\}_{\nu \in I}$ de $W$ par des domaines contractiles vérifiant la condition suivante: il existe une partition de $I$ en une collection finie de sous-ensembles $I_{\mu}(\mu=1, \cdots, r)$ telle que, pour chaque $\mu$, les domaines pour lesquels $\nu \in I_{\mu}$ soient deux à deux disjoints. Soit une partition différentiable de l'unité subordonnée au recouvrement et posons

$$
\tau_{\mu}=\sum_{\nu \in I_{\mu}} \varphi_{\nu}
$$

Soit $X$ un élément de $L^{*}$. Il existe une $(2 n-1)$-forme $\psi$ de $W$ telle que:

$$
\mu(X)=d u, \quad u \eta=d \psi .
$$

Posons $\psi_{\mu}=\tau_{\mu} \psi, u_{\mu} \eta=d \psi_{\mu}(\mu=1, \cdots, r)$. Pour $\mu$ fixé, considérons les domaines $\left\{U_{\nu}\right\}_{\nu \in I_{\mu}}$ deux à deux disjoints ; la fonction $\left.u_{\mu}\right|_{U_{\nu}}$ est telle que $\left.u_{\mu}\right|_{U_{\nu}} \eta=$ $d\left(\varphi_{\nu} \psi\right)$ donc appartient à $N_{1}$; en appliquant à $\left.u_{\mu}\right|_{U_{\nu}}$ le lemme de Calabi, on voit que $u_{\mu}$ est la somme des parenthèses de $2 n$ couples $\left(v_{(i)}, w^{(i)}\right)$ d'éléments de $N$, où $S\left(v_{(i)}\right), S\left(w^{(i)}\right) \subset \cup_{I_{\mu}} U_{\nu}$.

Si $X_{\mu}=\mu^{-1}\left(d u_{\mu}\right)$. on a $X_{\mu} \in\left[L^{*}, L^{*}\right]$ et $X$ somme finie d'élements de $\left[L^{*}, L^{*}\right]$ appartient à $\left[L^{*}, L^{*}\right]$. Il en résulte $\left[L^{*}, L^{*}\right]=L^{*}$. Comme

$$
[L, L] \subset L^{*}=\left[L^{*}, L^{*}\right] \subset[L, L]
$$

on a $[L, L]=L^{*}$. Nous énonçons

Proposition 1. Les algèbres $L$ et $L^{*}$ admettent $L^{*}$ pour idéal dérivé.

b) Soit maintenant $\left\{U_{\nu}\right\}_{\nu \in I}$ un recouvrement de $W$ par des domaines contractiles et soit $\left\{\varphi_{\nu}\right\}$ une partition différentiable de l'unité subordonnée.

Si $X$ est un élément de $L_{1}$, on a $\mu(X)=d u$, où $u \in N_{1}$. Il existe une $(2 n-1)$ forme $\psi$ à support $S(\psi)$ compact telle que $u \eta=d \psi$. Posons $\psi_{\nu}=\varphi_{\nu} \psi, u_{\nu} \eta=$ $d \psi_{\nu}$. La forme $\psi$ étant à support compact, il existe seulement un nombre fini de $\psi_{\nu}$, donc de $u_{\nu}$, qui sont non nuls. Pour $\nu$ fixé, la fonction $u_{\nu} \in N_{1}$ est à support $S\left(u_{\nu}\right) \subset U_{\nu}$; en lui appliquant le lemme de Calabi, on voit que $u_{\nu}$ est la somme des parenthèses de $2 n$ couples $\left(v_{(i)}, w^{(i)}\right)$ d'éléments de $N_{1}$.

Par suite $X_{\nu} \in\left[L_{1}, L_{1}\right]$ et $X$ somme finie d'éléments de $\left[L_{1}, L_{1}\right]$ appartient à $\left[L_{1}, L_{1}\right]$. Il en résulte $\left[L_{1}, L_{1}\right]=L_{1}$. Comme

$$
\left[L_{1}, L_{1}\right] \subset\left[L_{0}^{*}, L_{0}\right] \subset\left[L_{0}^{*}, L\right] \subset L_{1}
$$

on obtient immédiatement la proposition suivante:

Proposition 2. Les algèbres $L_{0}^{*}$ et $L_{1}$ admettent $L_{1}$ comme idéal dérivé. De plus: 


$$
L_{1}=\left[L_{1}, L_{0}\right]=\left[L_{0}^{*}, L_{0}\right]=\left[L^{*}, L_{0}\right]=\left[L_{1}, L\right]=\left[L_{0}^{*}, L\right] .
$$

c) Sur la variété symplectique $(W, F)$ appelons forme cohomologique symplectique la 2-forme $S$ sur $H_{0}^{1}(W, R)$ définie de la manière suivante: si $[\alpha]$ et [ $\beta$ ] sont deux éléments de $H_{0}^{1}(W ; R)$ admettant pour représentants les deux 1 -formes $\alpha, \beta$ fermées, à supports compacts, on a:

$$
S([\alpha],[\beta])=\int_{W} \Lambda(\alpha \wedge \beta) \eta .
$$

$S$ peut être triviale (c'est-à-dire identiquement nulle) ou non. On établit aisément:

Proposition 3. L'idéal dérivé de $L_{0}$ est soit $L_{1}$, soit $L_{0}^{*}$ selon que $S$ est triviale ou non.

\section{Fermés de nullité et idéaux canoniques associés}

Soit $P$ l'espace des $(2 n-1)$-formes à supports compacts de $W$. Dans la suite, nous introduisons sur $P$ (resp. sur $N_{1}$ ) la topologie usuelle qui intervient sur les formes tests $(2 n-1)$-formes ou 0 -formes en théorie des distributions. Nous définissons ainsi $P, N_{1}$ et par suite $L_{1}$ comme espaces vectoriels topologiques. L'application $\psi \in P \rightarrow u=-\delta * \psi \in N_{1}$ est continue. La topologie de $L_{1}$ (resp. $N_{1}$ ) est manifestement compatible avec le crochet de $L_{1}\left(\right.$ resp. $N_{1}$ ).

a) Soit $M$ un sous-espace vectoriel de $L$. Nous appelons fermé de nullité de $M$ et notons $n(M)$ l'ensemble fermé $f$ des points $x$ de $W$ tels que $X(x)=0$ pour tout $X$ de $M$; $\lceil f=\lceil n(M)$ est l'ouvert complémentaire. L'espace $M$ est dit transitif en $x_{0} \in\left\lceil f\right.$ si les valeurs en $x_{0}$ des éléments de $M$ engendrent l'espace vectoriel tangent en $x_{0}$ à $W ; M$ est transitif sur un ouvert de $W$ s'il est transitif en tout point de cet ouvert. Nous notons que $L_{1}\left(\right.$ et $\left.L_{0}^{*}, L_{0}, L^{*}, L\right)$ est transitive sur $W$.

Si $M \subset L_{1}$, son adhérence $\bar{M}$ dans $L_{1}$ a même fermé de nullité. En effet on a trivialement $n(\bar{M}) \subset n(M)$. D'autre part si $\mu^{-1}(d u) \subset \bar{M}$ on a, par passage à la limite, $\mu^{-1}(d u)=0$ sur $n(M)$. Ainsi $n(\bar{M})=n(M)$.

b) Etant donné un fermé $f$ de $W$, considérons l'espace des éléments $\psi$ de $P$ tels que $S(\psi) \subset \complement f$. A l'espace envisagé correspond par $\psi \rightarrow \mu^{-1}(-d \delta * \psi)$ $\in L_{1}$ un sous-espace vectoriel $I_{c}(f)$ de $L_{1}$. Si $f=\emptyset, I_{c}(f)=L_{1}$.

Si $X \in I_{c}(f), Y \in L$, à $[X, Y]$ on peut associer d'après (10.4) la $(2 n-1)$ forme $\psi_{w}=-*(u \mu(Y))$, où $\mu(X)=d u, u=-\delta * \psi_{u}$. On a $S\left(\psi_{w}\right) \subset S(u)$ $\subset S\left(\psi_{u}\right) \subset \complement f$. On voit ainsi que $I_{c}(f)$ est un idéal de $L$. Il en est de même pour $\overline{I_{c}(f)}$, d'après la continuité du crochet.

Ces idéaux admettent $f$ comme fermé de nullité. En effet il est clair que si $X \in I_{c}(f)$, on a $S(X) \subset \complement f$; ainsi $f \subset n\left(I_{c}(f)\right)$. D'autre part si $x_{0} \in \complement f$, on peut trouver un élément $\psi$ de $P$ vérifiant $S(\psi) \subset \complement f$ et tel que $d \delta * \psi$ soit $\neq 0$ en $x_{0}$; on a $\complement f \subset \complement n\left(I_{c}(f)\right)$. Ainsi $n\left(I_{c}(f)\right)=f$ et $n\left(\overline{I_{c}(f)}\right)=f$. 
Proposition. $I_{c}(f), \overline{I_{c}(f)}$ sont des idéaux de L admettant $f$ comme fermé de nullité; $I_{c}(f)$ est dit l'idéal canonique associé à $f$.

c) Nous notons $\hat{I}_{c}(f)$ l'idéal de $L$ associé à l'espace des éléments $\psi$ de $P$ tels que $S(\psi) \subset \overline{\complement f}=\complement \stackrel{\complement}{f} ; I_{d}^{1}(f)$ (resp. $I_{d}(f)$ est l'idéal de $L$ défini par les éléments $X$ de $L_{1}$ (resp. $L$ ) tels que $S(X) \subset \overline{\complement f}$. Si $X \in \overline{I_{c}(f)}$, on a $S(X) \subset \overline{\complement f}$ et il vient:

$$
\overline{I_{c}(f)} \subset I_{d}^{1}(f) \subset I_{d}(f) .
$$

Posons $\hat{f}=\overline{\dot{f}}$; on a $\dot{f} \subset \hat{f} \subset f$ et par suite $\complement f \subset \complement \hat{f} \subset \complement \dot{f}=\overline{\complement f}$. Les idéaux $\hat{I}_{c}(f)$, $I_{d}^{1}(f), I_{d}(f)$ admettent $\hat{f}$ comme fermé de nullité. Raisonnons sur $\hat{I}_{c}(f) ;$ de $S(\psi)$ $\subset \complement \mathfrak{f}$ on déduit que $\psi$ (resp. $X)$ s'annule sur $\dot{f}$, donc sur $\hat{f}$ et $\hat{f} \subset n\left(\hat{I}_{c}(f)\right)$. Inversement si $x_{0} \in \complement \hat{f}$, il existe $\psi \in P$ vérifiant $S(\psi) \subset \overline{\complement f}$, telle que si $X$ est le vecteur correspondant $X\left(x_{0}\right) \neq 0$. On a donc $\complement \hat{f} \subset \complement n\left(\hat{I}_{c}(f)\right)$.

Il résulte directement de (10.4), (10.5) que:

$$
\left[L^{*}, I_{d}^{1}(f)\right] \subset \hat{I}_{c}(f), \quad\left[L_{1}, I_{d}(f)\right] \subset \hat{I}_{c}(f) .
$$

Si $X \in \hat{I}_{c}(f)$, il existe un élément $\psi$ de $P$ à support compact $K=S(\psi) \subset \complement \stackrel{\complement}{f}$ tel que $X=\mu^{-1}(-d \delta * \psi) ; \psi$ s'annulant sur $\hat{f}$, on a $\hat{K} \subset \complement \hat{f}$. On peut construire une suite $\left\{\psi_{r}\right\}$ d'éléments de $P$ vérifiant $S\left(\psi_{r}\right) \subset K \subset \subset \hat{K} \hat{f}$ et qui converge vers $\psi$. Si $X_{r}=\mu^{-1}\left(-d \delta * \psi_{r}\right), X_{r} \in I_{c}(\hat{f})$ converge vers $X$ et l'on obtient :

$$
\hat{I}_{c}(f) \subset \overline{I_{c}(\hat{f})} .
$$

d) Nous nous proposons d'établir le lemme suivant:

Lemme. Si $M$ est un sous espace vectoriel de $L$ tel que $n(M)=f$ et si $M$ est invariant par $I_{c}(f)$, le sous-espace $\left[M, I_{c}(f)\right]$ invariant par $I_{c}(f)$ est transitif sur 〔f et admet $f$ pour fermé de nullité.

Comme $\left[M, I_{c}(f)\right] \subset M$, on a $\left[\left[M, I_{c}(f)\right], I_{c}(f)\right] \subset\left[M, I_{c}(f)\right]$ et $\left[M, I_{c}(f)\right]$ est bien invariant par l'idéal $I_{c}(f)$.

Si $x_{0} \in\left[f\right.$, il existe $X \in M$ tel que $X\left(x_{0}\right) \neq 0$, élément $X$ que nous fixons. Si $Y \in I_{c}(f)\left(\right.$ avec $\left.\mu(Y)=d v, v=-\delta * \psi_{v}\right)$, on a $\mu([X, Y])=d w$ avec $w=$ $i(X) d v$. Soit $U \subset \complement f$ un domaine contractile de coordonnées contenant $x_{0}$. Nous nous proposons de montrer que l'on peut choisir $v$ telle que $d i(X) d v$ prenne en $x_{0}$ une valeur imposée $\bar{\zeta}$.

L'indice 0 indiquera que les valeurs sont prises en $x_{0}$. On a en coordonnées locales

$$
\left\{\partial_{k}\left(X^{i} \partial_{i} v\right)\right\}_{0}=\bar{\zeta}_{k}
$$

soit:

$$
\left\{X^{i} \partial_{i k} v+\partial_{k} X^{i} \cdot \partial_{i} v\right\}_{0}=\bar{\zeta}_{k}
$$

Choississons $(d v)_{0}=\xi$, où $\xi$ est un covecteur donné en $x_{0}$. Il suffit alors de satis- 
faire la condition

$$
X_{0}^{i}\left(\partial_{i k} v\right)_{0}=\zeta_{k}
$$

où $\zeta$ est un covecteur donné en $x_{0}$.

Etant donnés en $x_{0}$ un vecteur $X_{0} \neq 0$ et un covecteur arbitraire $\zeta$, il existe en ce point un 2-tenseur symétrique $\tau$ tel que:

$$
i\left(X_{0}\right) \tau=\zeta .
$$

Pour le voir, adoptons un instant en $x_{0}$ un repère tel que $X_{0}^{1} \neq 0, X_{0}^{u}=0$ pour $u \neq 1$. La relation (13.4) s'écrit dans ce repère:

$$
X_{0}^{1} \tau_{1 k}=\zeta_{k},
$$

et il suffit d'introduire le 2-tenseur $\tau$ dont les composantes dans ce repère sont:

$$
\tau_{1 k}=\tau_{k 1}=\zeta_{k} / X_{0}^{1}, \quad \tau_{u v}=0 \quad \text { pour } u, v \neq 1 .
$$

Cela posé, on peut trouver une fonction $v$ à support compact $K \subset U$ telle qu'en $x_{0}$ :

$$
\left(\partial_{i} v\right)_{0}=\xi_{i}, \quad\left(\partial_{i k} v\right)_{0}=\tau_{i k} .
$$

En modifiant cette fonction dans $U-K$, on peut supposer que $v \in N_{1}$ avec $S(v) \subset U$.

Pour $Y=\mu^{-1}(d v), Z=[X, Y]=\mu^{-1}(d w)$ prend en $x_{0}$ une valeur arbitrairement donnée; il existe une $(2 n-1)$-forme $\psi_{v}$ correspondant à $v$, telle que $S\left(\psi_{v}\right) \subset U$ donc $\mu^{-1}(d v)=Y$ appartient bien à $I_{c}(f)$. Ainsi $\left[M, I_{c}(f)\right]$ est transitif sur $[f$ et, en particulier, admet $f$ pour fermé de nullité.

On notera que comme $S(v) \subset U$, on a $\mu(Z)=d w$, avec $S(w) \subset U$.

e) De ce lemme, on déduit le théorème suivant:

Théorème. Si $M$ est un sous-espace vectoriel de $L$ tel que $n(M)=f$ et si $M$ est invariant par $I_{c}(f)$, on a

$$
I_{c}(f) \subset M, \quad\left[M, I_{c}(f)\right]=I_{c}(f) .
$$

En particulier, $M \neq\{0\}$ ne peut être de dimension finie.

En effet si $x_{0} \in \llbracket f$, soit $U \subset \complement f$ un domaine contractile contenant $x_{0}$. Choisissons une base $\left(Z_{(i)}\right)_{0}$ de l'espace vectoriel tangent en $x_{0}$ à $W$; d'après le lemme, il existe $2 n$ éléments $Z_{(i)}$ de $\left[M, I_{c}(f)\right] \subset M$ prenant en $x_{0}$ les valeurs $\left(Z_{(i)}\right)_{0}$. En vertu de la remarque de la fin du $d$, nous pouvons supposer que les $2 n$ fonctions $w^{(i)} \in N_{1}$ pour lesquelles $\mu\left(Z_{(i)}\right)=d w^{(i)}$ ont leurs supports dans un compact $K \subset U$.

Il existe alors un domaine contractile $U^{\prime}$ contenant $x_{0}$, avec $\bar{U}^{\prime} \subset U$, tel que les $x^{i}=\left.w^{(i)}\right|_{U^{\prime}}$ définisseur sur $U^{\prime}$ un système de coordonnées locales. Soit $u$ 
un élément de $N_{1}$ tel que $S(u) \subset U^{\prime}$. D'après le lemme de Calabi généralisé, il existe $2 n$ fonctions $v_{(i)} \in N_{1}$, à supports dans $U$, telles que:

$$
u=\sum_{i}\left\{v_{(i)}, w^{(i)}\right\}
$$

soit.

Comme $S\left(v_{(i)}\right) \subset U$, il existe une $(2 n-i)$-forme $\psi_{(i)}$ correspondante, à support compact contenu dans $U$ et $\mu^{-1}\left(d v_{(i)}\right) \in I_{c}(f)$. Par suite $\mu^{-1}(d u) \in M$.

Soit maintenant $\psi$ une $(2 n-1)$-forme à support compact $S(\psi) \subset\lceil f$ et soit $u=-\delta * \psi$ l'élément correspondant de $N_{1}$. Introduisons un recouvrement fini convenable $\left\{U_{\nu}\right\}$ d'un voisinage ouvert de $S(\psi)$ et une partition $\left\{\varphi_{\nu}\right\}$ différentiable de l'unité subordonnée. Si on pose $\psi_{\nu}=\varphi_{\nu} \psi, u_{\nu} \eta=d \psi_{\nu}$, chaque élément $\mu^{-1}\left(d u_{\nu}\right)$ appartient à $M$ et $X=\mu^{-1}(d u)$, somme finie d'élements de $M$, appartient à $M$. On a donc $I_{c}(f) \subset M$ et $I_{c}(f)$ étant de dimension infinie pour $f \neq W, M$ est de dimension infinie.

L'espace $\left[M, I_{c}(f)\right]$ admet $f$ comme fermé de nullité et est invariant par $I_{c}(f)$. Il résulte de la première partie et du fait que $I_{c}(f)$ est un idéal de $L$ :

$$
I_{c}(f) \subset\left[M, I_{c}(f)\right] \subset I_{c}(f),
$$

ce qui entraîne bien:

$$
\left[M, I_{c}(f)\right]=I_{c}(f)
$$

et le théorème démontré.

Pour $f=\emptyset$, on a $I_{c}(f)=L_{1}$. Il résulte du théorème:

Corollaire. Tout sous-espace vectoriel $M$ de $L$ invariant par $L_{1}$ et tel que $n(M)=\emptyset$ vérifie

$$
L_{1} \subset M, \quad\left[M, L_{1}\right]=L_{1} \text {. }
$$

\section{Idéaux et idéaux canoniques-Semi-simplicité}

a) Un fermé $f$ de $W$ étant donné, prenons $M=I_{c}(f)$ idéal de $L$. On a:

$$
\left[I_{c}(f), I_{c}(f)\right]=I_{c}(f)
$$

On en déduit par passage à la limite:

$$
\left[\overline{I_{c}(f)}, \overline{I_{c}(f)}\right]=\overline{I_{c}(f)}
$$

Comme $I_{d}^{1}(f)$ admet $\hat{f}$ comme fermé de nullité, on a $\left[I_{c}(\hat{f}), I_{d}^{1}(f)\right]=I_{c}(\hat{f})$ et par passage à la limite $\left[\overline{I_{c}(\hat{f})}, I_{d}^{1}(f)\right]=\overline{I_{c}(\hat{f})}$. Ainsi d'après $(13.1) \overline{I_{c}(\hat{f})} \subset \hat{I}_{c}(f)$ et il résulte de (13.2) que $I_{c}(\hat{f})=\hat{I}_{c}(f)$. Nous énonçons :

Proposition 1. Si f est un fermé de $W$, et si $\hat{f}=\bar{f}$, on a 


$$
\left[I_{c}(f), I_{c}(f)\right]=I_{c}(f), \quad\left[\overline{I_{c}(f)}, \overline{I_{c}(f)}\right]=\overline{I_{c}(f)}, \quad \overline{I_{c}(\hat{f})}=\hat{I}_{c}(f) .
$$

Nous abandonnons dans la suite la notation $\hat{I}_{c}(f)$.

b) D'après le corollaire du $\S 13$, tout idéal $A$ de $L$ tel que $n(A)=\emptyset$ contient $L_{1}$, ce qui peut être considéré comme une généralisation d'un résultat de Calabi. Dans la suite, $A$ désignera une algèbre contenant $L_{1}$ supposée donnée. Prenons pour $M$ un idéal $I$ de $A$ tel que $n(I)=f ; I$ est invariant par $L_{1}$, donc par $I_{c}(f)$. On déduit du théorème du $\S 13$ :

Proposition 2. Si I est un idéal de A tel que $n(I)=f$, on $a$ :

$$
I_{c}(f) \subset I, \quad\left[I, I_{c}(f)\right]=I_{c}(f) .
$$

En particulier $I \neq\{0\}$ ne peut être de dimension finie et $I$ est transitif sur 〔f.

Soit $\bar{I}$ un idéal fermé de $L_{1}$ tel que $n(\bar{I})=f$. On a d'après la proposition 2

$$
\overline{I_{c}(f)} \subset \bar{I}, \quad\left[\bar{I}, \overline{I_{c}(f)}\right]=\overline{I_{c}(f)} .
$$

De $\bar{I} \subset I_{d}^{1}(f)$, on déduit d'après (13.1) que $\left[\bar{I}, L^{*}\right] \subset \overline{I_{c}(\hat{f})}$.

Supposons $f=\hat{f}$. Comme:

$$
\overline{I_{c}(f)}=\left[\bar{I}, \overline{I_{c}(f)}\right] \subset[\bar{I}, \bar{I}] \subset\left[\bar{I}, L_{1}\right] \subset\left[\bar{I}, L^{*}\right] \subset \overline{I_{c}(\hat{f})}=\overline{I_{c}(f)}
$$

les inclusions sont toutes des égalités.

Inversement supposons que $\bar{I}$ soit un sous-espace fermé de $L_{1}$ (avec $n(\bar{I})=$ $f ; f=\hat{f})$ contenant $\overline{I_{c}(f)}$. Il résulte de (13.1) que comme $\bar{I} \subset I_{d}^{1}(f), \bar{I}$ est invariant par $L_{1}$, donc est un idéal de $L_{1}$. Ainsi :

Proposition 3. Pour qu'un sous-espace fermé $\bar{I}$ de $L_{1}$ tel que $n(\bar{I})=f=\bar{f}$ soit un idéal de $L_{1}$, il faut et il suffit que

$$
\overline{I_{c}(f)} \subset \bar{I} .
$$

S'il en est ainsi, on a:

$$
\left[\bar{I}, \overline{I_{c}(f)}\right]=[\bar{I}, \bar{I}]=\left[\bar{I}, L_{1}\right]=\left[\bar{I}, L^{*}\right]=\overline{I_{c}(f)} .
$$

En particulier, $\bar{I}$ est un idéal de $L$.

c) Soit $I$ un idéal de $A$ tel que $n(I)=f, J$ un idéal de $I$ tel que $n(J)=f^{\prime}$, où nécessairement $f \subset f^{\prime}$. D'après $\left\lceil f^{\prime} \subset \complement f\right.$, on a $I_{c}\left(f^{\prime}\right) \subset I_{c}(f)$. D'après la proposition $2, J$ est invariant par $I_{c}(f)$, donc par $I_{c}\left(f^{\prime}\right)$ et le théorème du $\S 13$ s'applique à $J$. Il vient

Proposition 4. Si J est un idéal d'un idéal I de A tel que $n(J)=f^{\prime}$, on a:

$$
I_{c}\left(f^{\prime}\right) \subset J, \quad\left[J, I_{c}\left(f^{\prime}\right)\right]=I_{c}\left(f^{\prime}\right) .
$$

En particulier $J \neq\{0\}$ ne peut être de dimension finie et $J$ est transitif sur $\left\lceil f^{\prime}\right.$. 
Soit $\bar{J}$ un sous-espace fermé de $L_{1}$ tel que $n(\bar{J})=f^{\prime}=\hat{f}^{\prime}$, et qui est un idéal d'un idéal $I$ de $A$. On a d'après la proposition précédente $\overline{I_{c}\left(f^{\prime}\right)} \subset \bar{J}$. Il résulte de la proposition 3 que $\bar{J}$ est un idéal fermé de $L_{1}$ et les autres résultats de cette proposition s'appliquent à $\bar{J}$.

Proposition 5. Si $\bar{J}$, sous-espace fermé de $L_{1}$ tel que $n(\bar{J})=f^{\prime}=\overline{f^{\prime}}$ est un idéal d'un idéal de $I$ de $A, \bar{J}$ est un idéal fermé de $L_{1}$, donc un idéal de $L^{*}$.

d) Soit $I$ un idéal de $A, J$ un idéal abélien de $I$ (avec $n(J)=f^{\prime}$ ). On a d'après la proposition 4

$$
I_{c}\left(f^{\prime}\right) \subset J
$$

$J$ étant abélien:

$$
\left[J, I_{c}\left(f^{\prime}\right)\right]=\{0\}
$$

et il résulte de la proposition 4 :

$$
I_{c}\left(f^{\prime}\right)=\{0\} .
$$

Ainsi $f^{\prime}=W$ et $J=\{0\}$. On obtient:

Théorème. Tout idéal I de A est semi-simple. En particulier $L, L^{*}, L_{0}, L_{0}^{*}$, $L_{1}$ et tous leurs idéaux sont semi-simples.

\section{Centralisateur d'un idéal-Idéaux supplémentaires}

Soit $I$ un idéal de $A, Z(I)$ le centralisateur de $I$ dans $A$. L'idéal $Z(I) \cap I$ de $A$ est abélien, donc nul et l'on a:

$$
Z(I) \cap I=\{0\} .
$$

a) Nous allons établir la proposition suivante:

Proposition. Soit I un idéal de A tel que $n(I)=f$. Le centralisateur $Z(I)$ de I dans $A$ coïncide avec l'ensemble des éléments de $A$ qui s'annulent en tout point de $\overline{\complement f}$.

En effet, désignons par $I^{\prime}$ l'ensemble de ces éléments; l'idéal $I$ étant invariant par $L_{1}$, est invariant par $I_{c}(f)$ donc

$$
I_{c}(f) \subset I .
$$

Soit $X \in I_{c}(f)$ et $Z \in Z(I)$. D'après le $\S 8, \mathrm{~b},[Z, X]=0$ se traduit par

$$
i(X) \mu(Z)=0 .
$$

$I_{c}(f)$ étant transitif sur $\lceil f$, il résulte de (15.2) que $Z$ s'annule en tout point de $\left\lceil f\right.$, donc en tout point de $\overline{\complement f}$. Ainsi $Z(I) \subset I^{\prime}$.

Inversement soit $X \in I, Y \in I^{\prime}$. On a localement 


$$
[X, Y]^{i}=X^{r} \partial_{r} Y^{i}-Y^{r} \partial_{r} X^{i} .
$$

On voit sur (15.3), d'après les propriétés de $Y$, que $\left.[X, Y]\right|_{c f}=0$, donc que

$$
\left.[X, Y]\right|_{\overline{c f}}=0 .
$$

Soit $x \in f \mathfrak{f}$ un point intérieur à $f$. On voit sur (15.3), d'après les propriétés de $x$, que $[X, Y](x)=0$. Donc

$$
\left.[X, Y]\right|_{f} ^{\circ}=0 .
$$

Ainsi $[X, Y]=0$ sur $W$ et $Y \in Z(I)$. On a donc aussi $I^{\prime} \subset Z(I)$, ce qui démontre la proposition.

b) Nous allons établir le théorème suivant:

Théorème. Un idéal I non trivial de $A$ ne peut admettre un idéal supplémentaire dans $A$. Il en est ainsi en particulier pour $A=L, L^{*}, L_{0}, L_{0}^{*}, L_{1}$.

Supposons que $I$ admette un idéal $B$ supplémentaire de $I$ dans $A$. On a

$$
A=I \oplus B
$$

avec $[B, I]=\{0\}$, donc $B \subset Z(I)$. En décomposant $Z(I)$ selon (15.4), il vient:

$$
Z(I)=J \oplus B \quad(J \subset I) .
$$

Comme $Z(I) \cap J=\{0\}$, on a $J=\{0\}$ et $B=Z(I)$. Ainsi

$$
A=I \oplus Z(I) .
$$

Tout élément de $A$ s'annule sur $f \cap \overline{C f}$ (où $f=n(I)$ ). Mais $A$ contient $L_{1}$ transitif sur $W$; donc

$$
f \cap \overline{\complement f}=\emptyset .
$$

$W$ étant connexe, (15.5) entraîne que l'un des fermés $f$ ou $\overline{\complement f}$ est nécessairement vide. Si $f=\emptyset, Z(I)=(0)$ et $I=A$. Si $\overline{\complement f}=\emptyset, f=W$ et $I=\{0\}$. Dans les deux cas, I est un idéal trivial de $A$.

\section{DÉRIVATIONS ET PREMIERS GROUPES DE COHOMOLOGIE}

16. Caractère local des dérivations de $L, L^{*}$ et $N_{1}$

Nous vous proposons, au cours de cette section, de déterminer les dérivations des algèbres de Lie $L, L^{*}, N$ et $N_{1}$ [2] Une dérivation de l'algèbre de 
Lie $L$ est une application linéaire $D: L \rightarrow L$ telle que pour tout $Y, Z \in L$, on ait:

$$
D[Y, Z]=[D Y, Z]+[Y, D Z]
$$

Mêmes définitions pour une dérivation $D^{*}$ de $L^{*}, \mathscr{D}$ de $N, \mathscr{D}_{1}$ de $N_{1}$ ou $\mathscr{D}$ de $N / R$. On rappelle $(\S 4)$ que $N / R$ est isomorphe à $L^{*}$.

Nous allons d'abord établir le caractère local des dérivations de $L, L^{*}$ et $N_{1}$.

a) Soit $D$ une dérivation de $L, Y$ un élément de $L$. Supposons que, sur un ouvert $U$ de $W$, on ait $\left.Y\right|_{U}=0$; si $x \in U$ on peut trouver $Z \in L_{1}$ à support $K \subset U$, tel que $Z(x)$ soit un vecteur arbitrairement donné en $x$. On a $[Y, Z]$ $=0$, donc $D[Y, Z]=0$; d'autre part $[Y, D Z]_{U}=0$, donc, d'après $(16.1)$, $[D Y, Z]_{U}=0$. Ainsi $[D Y, Z]=0$, soit:

$$
d \Lambda(\mu(D Y) \wedge \mu(Z))=0, \quad \Lambda(\mu(D Y) \wedge \mu(Z))=C=\text { const } .
$$

Le premier membre étant à support compact inclus dans $K$, on a $C=0$ et par suite:

$$
i(Z) \mu(D Y)=0 \text {. }
$$

(16.2) étant valable en $x$ pour un vecteur arbitraire $Z(x)$, on a $(D Y)(x)=0$, donc $\left.D Y\right|_{U}=0$. Le même raisonnement s'applique aux dérivations de $L^{*}$. Ainsi

Proposition 1. Toute dérivation de $L$ (resp. $L^{*}$ ) est un opérateur local.

Pour des dérivations de $N$ ou $N_{1}$, on établit de même que pour $u \in N$, $u_{1} \in N_{1}$ :

$$
S\left(d \mathscr{D}_{1} u_{1}\right) \subset S\left(d u_{1}\right)
$$

Considérons en particulier la fonction $u=1$ de $N$; on a $d \mathscr{D} 1=0$ sur $W$ connexe. Ainsi si $\mathscr{D}$ est une dérivation de $N$, on a $\mathscr{D} 1=$ const. sur $W$.

Si $\overline{\mathscr{D}}$ est une dérivation de $N / R$, on a aussi pour $\bar{u} \in N / R$

$$
\mathrm{S}(d \overline{\mathscr{D}} \bar{u}) \subset S(d \bar{u}) .
$$

b) Soit $D$ une dérivation de $L$. Nous avons établi (§12) que $L^{*}=[L, L]$. Or d'après (16.1):

$$
D[L, L] \subset[L, L]
$$

Ainsi la restriction à $L^{*}$ d'une dérivation $D$ de $L$ est une dérivation $D^{*}$ de $L^{*}$. Inversement, soit $D^{*}$ une dérivation de $L^{*}$; si $Y \in L$ introduisons $Z \in L^{*}$ tel que $\left.Y\right|_{U}=\left.Z\right|_{U}$ sur un domaine contractile $U$ de $W$. D'après son caractère local, toute dérivation $D$ dont la restriction à $L^{*}$ coïncide avec $D^{*}$ est telle que $\left.D Y\right|_{U}=\left.D^{*} Z\right|_{U}$. D'après le caractère local de $D^{*}$, on définit ainsi une dériva- 
tion $D$ nécessairement unique de $L$ dont la restriction à $L^{*}$ coïncide avec $D^{*}$. Nous pouvons énoncer.

Proposition 2. L'espace des dérivation de $L^{*}$ est l'espace des restrictions à $L^{*}$ des dérivations de $L$. Il est isomorphe à l'espace des dérivations de $L$.

c) Nous allons établir

Proposition 3. Toute dérivation $\mathscr{D}_{1}$ de $N_{1}$ est une opérateur local.

En effet soit $U$ un domaine contractile. Si $u \in N_{1}$ est à support dans $U$, il résulte du lemme de Calabi qu'il existe $2 n$ couples $\left(v_{(i)}, w^{(i)}\right)$ d'éléments de $N_{1}$ à supports dans $U$ tels que:

$$
u=\sum_{i}\left\{v_{(i)}, w^{(i)}\right\}
$$

Il vient:

$$
\mathscr{D}_{1} u=\sum_{i}\left\{\mathscr{D}_{1} v_{(i)}, w^{(i)}\right\}+\sum_{i}\left\{v_{(i)}, \mathscr{D}_{1} w^{(i)}\right\}
$$

où chaque terme du second membre est à support dans $U$. Ainsi $S\left(\mathscr{D}_{1} u\right) \subset U$.

Cela posé soit $u$ un élément de $N_{1}$; il existe une $(2 n-1)$-forme $\psi$ à support compact $S(\psi)=K$ telle que $u \eta=d \psi$. Introduisons un recouvrement fini $\left\{U_{\nu}\right\}$ par des domaines contractiles d'un voisinage ouvert de $K$ et soit $\left\{\varphi_{\nu}\right\}$ une partition différentiable de l'unité subordonnée. Posons $\psi_{\nu}=\varphi_{\nu} \psi, u_{\nu} \eta \in d \psi_{\nu}$, où $u_{\nu} \in N_{1}$ et $S\left(u_{\nu}\right) \subset U_{\nu}$. On a $\mathscr{D}_{1} u=\sum \mathscr{D}_{1} u_{\nu}$ où $S\left(\mathscr{D}_{1} u_{\nu}\right) \subset U_{\nu}$. Par suite $S\left(\mathscr{D}_{1} u\right) \subset \cup U_{\nu}$. Ainsi

$$
S\left(\mathscr{D}_{1} u\right) \subset S(\psi)
$$

Soit $V$ un domaine contractile tel que $\left.u\right|_{V}=0$; on a $\left.d \psi\right|_{V}=d\left(\left.\psi\right|_{V}\right)=0$ et il existe sur $V$ une $(2 n-2)$-forme $\alpha_{\nu}$ telle que $\left.\psi\right|_{V}=d \alpha_{V}$. Donnons-nous une $(2 n-2)$-forme $\beta$ de $W$ à support compact telle que $\left.\beta\right|_{V}=\alpha_{V}$ et substituons à $\psi$ la forme $\bar{\psi}=\psi-d \beta$; on a $\left.\bar{\psi}\right|_{V}=0$ et $u \eta=d \bar{\psi}$. Comme $S\left(\mathscr{D}_{1} u\right) \subset S(\bar{\psi})$, il vient $\left.\mathscr{D}_{1} u\right|_{V}=0$ et $\mathscr{D}_{1}$ est un opérateur local.

d) Soit $\mathscr{D}$ une dérivation de $N$. Etudions sa restriction à $N_{1}$. Si $u \in N_{1}$ est à support dans le domaine contractile $U$, on a (16.4) et il vient:

$$
\mathscr{D} u=\sum_{i}\left\{\mathscr{D} v_{(i)}, w^{(i)}\right\}+\sum_{i}\left\{v_{(i)}, \mathscr{D} w^{(i)}\right\}
$$

Comme $v_{(i)}, w^{(i)} \in N_{1}$, chaque terme du second membre appartient à $N_{1}$ et $\mathscr{D} u \in N_{1}$.

Si $u$ est un élément quelconque de $N_{1}$, on voit comme au $c$ que $\mathscr{D} u$ est somme finie d'éléments $\mathscr{D} u_{\nu}$, de $N_{1}$, donc $\mathscr{D} u$ appartient à $N_{1}$.

Ainsi la restriction de $\mathscr{D}$ à $N_{1}$ est une dérivation $\mathscr{D}_{1}$ de $N_{1}$. Inversement, soit $\mathscr{D}_{1}$ une dérivation de $N_{1}$; si $u \in N$ introduisons $u_{1} \in N_{1}$ telle que $\left.u\right|_{U}=\left.u_{1}\right|_{U}$ pour un domaine $U$ et posons $\left.\mathscr{D} u\right|_{U}=\left.\mathscr{D}_{1} u_{1}\right|_{U}$. D'après le caractère local de $\mathscr{D}_{1}$, on définit ainsi une dérivation $\mathscr{D}$ de $N$ de càractère local dont la restriction à 
$N_{1}$ coïncide avec $\mathscr{D}_{1}$. Si $\mathscr{D}$ est une dérivation locale de $N$ dont la restriction à $N_{1}$ est nulle, on a nécessairement $\left.\mathscr{D} u\right|_{U}=0$ pour tout $u \in N$ et tout domaine $U$, donc $\mathscr{D}=0$. Ainsi

Proposition 4. L'espace des dérivations de $N_{1}$ est l'espace des restrictions à $N_{1}$ des dérivations de $N$. Il est isomorphe à l'espace des dérivations locales de $N$.

\section{Détermination des dérivations locales de $N$}

a) Proposons-nous de déterminer les dérivations locales de $N$. A cet effet nous utilisons le lemme suivant

Lemme. Si $\mathscr{D}$ est une dérivation de $N$, on a pour tout $u, v \in N$ :

$$
\mathscr{D}\left\{u^{2}, v\right\}=\left\{\mathscr{D} u^{2}-2 u \mathscr{D} u, v\right\}+2 u \mathscr{D}\{u, v\}+2 \mathscr{D} u\{u, v\} \text {. }
$$

En effet:

$$
\mathscr{D}\left\{u^{2}, v\right\}=\left\{\mathscr{D} u^{2}, v\right\}+\left\{u^{2}, \mathscr{D} v\right\}
$$

Or:

$$
\left\{u^{2}, \mathscr{D} v\right\}=2 u\{u, \mathscr{D} v\}=2 u \mathscr{D}\{u, v\}-2 u\{\mathscr{D} u, v\}
$$

où l'on $\mathrm{a}$ :

$$
\begin{aligned}
u\{\mathscr{D} u, v\} & =\Lambda(d(u \mathscr{D} u) \wedge d v)-\mathscr{D} u \Lambda(d u \wedge d v) \\
& =\{u \mathscr{D} u, v\}-\mathscr{D} u\{u, v\}
\end{aligned}
$$

Il en résulte:

$$
\left\{u^{2}, \mathscr{D} v\right\}=2 u \mathscr{D}\{u, v\}+2 \mathscr{D} u\{u, v\}-2\{u \mathscr{D} u, v\},
$$

ce qui entraîne (17.1).

b) Cela posé soit $\mathscr{D}$ une dérivation locale de $N$ et $u$ un élément de $N$. Si $x$ est un point où $(d u)_{x} \neq 0$ on peut trouver une carte canonique $\left(x^{\alpha}, x^{\alpha}\right)(\alpha=1$, $\cdots, n ; \bar{\alpha}=\alpha+n ; x^{i} \in N$ où $\left.i=1, \cdots, 2 n\right)$ dont le domaine $U$ contient $x$ et qui est telle que $\left.x^{1}\right|_{U}=\left.u\right|_{U}$ (Elie Cartan). On a sur $U$

$$
\{u, v\}_{U}=\partial_{\overline{1}} v, \quad\left\{u^{2}, v\right\}=2 u\{u, v\} .
$$

Pour $v=x^{\alpha}$ ou $v=x^{\alpha}(\operatorname{avec} \bar{\alpha} \neq \overline{1})$, on a donc:

$$
\{u, v\}_{U}=0, \quad\left\{u^{2}, v\right\}_{U}=0 .
$$

Pour $v=x^{\overline{1}}$ :

$$
\{u, v\}_{U}=1, \quad\left\{u^{2}, v\right\}_{U}=\left.2 u\right|_{U}
$$


Il résulte donc de (17.1) appliquée à $v=x^{\alpha}, x^{\bar{\alpha}}(\operatorname{avec} \bar{\alpha} \neq \overline{1})$ que l'on a sur $U$ :

$$
\partial_{i}\left(\mathscr{D} u^{2}-2 u \mathscr{D} u\right)=0, \quad i \neq 1 .
$$

Posons $\mathscr{D} 1=K$. Pour $v=x^{\overline{1}}$, il vient sur $U$ d'après (17.1)

$$
2 \mathscr{D} u=\partial_{1}\left(\mathscr{D} u^{2}-2 u \mathscr{D} u\right)+2 K u+2 \mathscr{D} u .
$$

soit:

$$
\partial_{1}\left(\mathscr{D} u^{2}-2 u \mathscr{D} u+K u^{2}\right)=0
$$

Ainsi au point $x$ on a $\left[d\left(\mathscr{D} u^{2}-2 u \mathscr{D} u+K u^{2}\right)\right]_{x}=0$. Si $A$ est l'ouvert défini par les points de $W$ où $d u \neq 0$, on a sur $\bar{A}$ :

$$
d\left(\mathscr{D} u^{2}-2 u \mathscr{D} u+K u^{2}\right)=0 .
$$

Si $\llbracket A$ possède des points intérieurs $y$, il existe un voisinage ouvert $V$ de $y$ tel que $\left.d u\right|_{V}=0,\left.d u^{2}\right|_{V}=0$ donc, d'après $\S 16$, a, tel que $\left.d \mathscr{D} u\right|_{V}=0,\left.d \mathscr{D} u^{2}\right|_{V}$ $=0$. On voit que (17.2) est satisfaite sur $W$ connexe, et que par suite:

$$
\mathscr{D} u^{2}-2 u \mathscr{D} u+K u^{2}=C=\text { const. }
$$

$\mathscr{D}$ étant de caractère local, en substituant à $u$ une fonction $u^{\prime} \in N$ qui coïncide avec $u$ sur un ouvert et qui est nulle sur un autre ouvert, on a $C=0$. Aussi, pour tout $u \in N: D u^{2}-2 u \mathscr{D} u+K u^{2}=0$, et pour tout $u, v \in N$, il vient par polarité :

$$
\begin{aligned}
& \mathscr{D}(u v)-u \mathscr{D} v-v \mathscr{D} u+K u v \\
& \quad=(\mathscr{D}-K)(u v)-u(\mathscr{D}-K) v-v(\mathscr{D}-K) u=0 .
\end{aligned}
$$

$(\mathscr{D}-K)$ définit donc un champ de vecteurs $X$ de $W$ et si $\mathscr{L}(X)$ est la dérivation de Lie correspondante, ou a $\mathscr{D}=\mathscr{L}(X)+K$. Pour que $\mathscr{D}$ soit une derivation de $N$, il faut et il suffit que:

$$
\begin{aligned}
\mathscr{L}(X) \cdot \Lambda(d u \wedge d v) & -\Lambda(\mathscr{L}(X) d u \wedge d v) \\
& -\Lambda(d u \wedge \mathscr{L}(X) d v)-K \Lambda(d u \wedge d v)=0
\end{aligned}
$$

soit, pour tout $u, v \in N$

$$
(\mathscr{L}(X) \Lambda-K \Lambda)(d u \wedge d v)=0 .
$$

Ainsi $\mathscr{D}=\mathscr{L}(X)+K$ est une dérivation si et seulement si

$$
\mathscr{L}(X) \Lambda-K \Lambda=0 \quad \text { ou } \quad \mathscr{L}(X) F^{-1}-K F^{-1}=0,
$$

c'est-à-dire si 


$$
\mathscr{L}(X) F+K F=0,
$$

et $X$ est une t.i. conforme symplectique de la variété (voir $\S 5$ ). On obtient:

Théorème. L'algèbre de Lie naturelle des dérivations locales de $N$ est isomorphe à l'algèbre de Lie $L^{c}$ des transformations infinitésimales conformes symplectiques de $(W, F)$ par l'isomorphisme défini de la manière suivante: si $X \in L^{c}$, on a $\mathscr{L}(X) F+K_{X} F=0$ et $X$ donne la dérivation

$$
\mathscr{D}_{X}=\mathscr{L}(X)+K_{X}
$$

\section{Détermination des dérivations de $L^{*}, L$ et $L^{c}$}

a) Soit $\mathscr{D}$ une dérivation de $N$. A partir de $\mathscr{D}$, on peut définir par $\overline{\mathscr{D}} \bar{u}=$ $\overline{\mathscr{D}} \mathrm{u}$ une dérivation $\overline{\mathscr{D}}$ de $N / R$ telle que $\overline{\mathscr{D}} \circ \pi=\pi \circ \mathscr{D}$ (notations du $\S 4$, a).

Soit $\overline{\mathscr{D}}$ une dérivation de $N / R$. Cherchons une application linéaire $\mathscr{D}_{1}: N_{1} \rightarrow$ $N_{1}$ telle que l'on ait $d \mathscr{D}_{1} u_{1}=d \overline{\mathscr{D}} \bar{u}_{1}$ pour tout $u_{1} \in N_{1}$. Une telle application est nécessairement unique, car $\mathscr{D}_{1} u_{1}=C$ n'appartient à $N_{1}$ que pour $C=0 ; \overline{\mathscr{D}}$ étant une dérivation, on en déduit que:

$$
\mathscr{D}_{1}\left\{u_{1}, v_{1}\right\}-\left\{\mathscr{D}_{1} u_{1}, v_{1}\right\}-\left\{u_{1}, \mathscr{D}_{1} v_{1}\right\}=C_{1}=\text { const. }
$$

Le premier membre appartenant à $N_{1}$, on a $C_{1}=0$ et $\mathscr{D}_{1}$, si elle existe, est une dérivation de $N_{1}$.

Cela posé soit $U$ un domaine contractile. Si $u \in N_{1}$ est à support dans $U$, on pose par définition

$$
\mathscr{D}_{1} u=\sum\left\{\overline{\mathscr{D}}_{(i)}, \bar{w}^{(i)}\right\}+\sum\left\{\bar{v}_{(i)}, \overline{\mathscr{D}} \bar{w}^{(i)}\right\} \in \overline{\mathscr{D}} \bar{u}
$$

où les notations sont celles du $\S 16, c$. Les termes du second membre appartiennent à $N_{1}$. Ainsi $\mathscr{D}_{1} u \in N_{1}, S\left(\mathscr{D}_{1} u\right) \subset U$ et $d \mathscr{D}_{1} u=d \overline{\mathscr{D}} \bar{u}$.

Soit $u$ un élément arbitraire de $N_{1}$. Avec le même recouvrement qu'au $\S 16$, c, posons $\mathscr{D}_{1} u=\sum \mathscr{D}_{1} u_{\nu}$ où les $\mathscr{D}_{1} u_{\nu}$ sont définis comme ci-dessus; on a $\mathscr{D}_{1} u \epsilon$ $N_{1}$ et $d \mathscr{D}_{1} u=\sum d \overline{\mathscr{D}} \bar{u}_{\nu}=d \overline{\mathscr{D}} \bar{u}$.

La dérivation $\mathscr{D}_{1}$ de $N_{1}$ ainsi construite est la restriction à $N_{1}$ d'une dérivation locale $\mathscr{D}$ de $N$. Si $u \in N$, introduisons $u_{1} \in N_{1}$ telle que $\left.u\right|_{U}=\left.u_{1}\right|_{U}$ pour un domaine $U$. On a

$$
\left.d \mathscr{D} u\right|_{U}=\left.d \mathscr{D}_{1} u_{1}\right|_{U}=\left.d \overline{\mathscr{D}} \bar{u}_{1}\right|_{U}=\left.d \overline{\mathscr{D}} \bar{u}\right|_{U}
$$

d'après le caractère local de $d \overline{\mathscr{D}}$ traduit par (16.3). Ainsi $\overline{\mathscr{D} u}=\overline{\mathscr{D}} \bar{u}$ et $\mathscr{D}$ est telle que

$$
\pi \circ \mathscr{D}=\overline{\mathscr{D}} \circ \pi
$$

La dérivation locale $\mathscr{D}$ de $\mathrm{N}$ jouissant de la propriété (18.1) est unique. En 
effet si $\overline{\mathscr{D} u}=0$ pour tout $u \in N$, on a $\mathscr{D} u=C=$ const. ; en substituant à $u$ une fonction $u^{\prime} \in N$ qui coïncide avec $u$ sur un ouvert et qui est nulle sur un autre ouvert, on obtient $C=0$ et pour tout $u \in N, \mathscr{D} u=0$, donc $\mathscr{D}=0$.

Ainsi, d'après le théorème du $\S 17$, à toute dérivation de $N / R$ correspond une t.i. conforme symplectique unique $X$ telle que la dérivation s'écrive:

$$
\overline{\mathscr{D}}_{X}=\mathscr{L}(X)+K_{X} .
$$

b) L'algèbre de Lie $N / R$ est isomorphe à $L^{*}$ par l'isomorphisme $\sigma: \bar{v} \epsilon$ $N / R \rightarrow Y=\mu^{-1}(d \bar{v}) \in L^{*} ; \sigma$ définit par:

$$
\sigma \circ \overline{\mathscr{D}}=D^{*} \circ \sigma
$$

un isomorphisme de l'espace des dérivations de $N / R$ sur l'espace des dérivations de $L^{*}$.

Si $X \in L^{c}$, il vient :

$$
\sigma\left\{\left(\mathscr{L}(X)+K_{X}\right) \bar{v}\right\}=\mu^{-1}\left\{d\left(\mathscr{L}(X)+K_{X}\right) \bar{v}\right\}=\mu^{-1}\left\{\left(\mathscr{L}(X)+K_{X}\right) d \bar{v}\right\} .
$$

Or si $\alpha$ est une 1 -forme:

$$
\mathscr{L}(X) \mu^{-1}(\alpha)=\left(\mathscr{L}(X) \mu^{-1}\right) \alpha+\mu^{-1}(\mathscr{L}(X) \alpha)=\mu^{-1}\left\{\left(\mathscr{L}(X)+K_{X}\right) \alpha\right\} .
$$

Ainsi:

$$
\sigma\left\{\left(\mathscr{L}(X)+K_{X}\right) \bar{v}\right\}=\mathscr{L}(X) \mu^{-1}(d \bar{v})=\mathscr{L}(X) Y .
$$

On en déduit que si $D^{*}$ est une dérivation de $L^{*}$, il existe un élément $X$ de $L^{c}$ tel que $D^{*}$ soit donné par $D^{*}: Y \in L^{*} \rightarrow \mathscr{L}(X) Y \in L^{*}$. De même, d'après la proposition 2 du $\S 17$, si $D$ est une dérivation de $L$, il existe un élément $X$ de $L^{c}$ tel que $D$ soit donné par $D: Y \in L \rightarrow \mathscr{L}(X) Y \in L$. Nous énonçons

Théorème 1. Toute dérivation de $L^{*}$ (resp. L) est une transformation infinitésimale conforme symplectique $Y \rightarrow[X, Y]$, où $X \in L^{c}$.

c) Proposons-nous de déterminer les dérivations $D^{c}$ de $L^{c}$. Si $F$ n'est pas exacte $L^{c}=L$. Nous supposons donc $F$ exacte et par suite $W$ non compacte. D'après la proposition $2 \mathrm{du} \S 5$, on a $\left[L^{c}, L^{c}\right]=L$. Si $D^{c}$ est une dérivation de $L^{c}$, il résulte de la définition des dérivations que:

$$
D^{c}\left[L^{c}, L^{c}\right]=\left[D^{c} L^{c}, L^{c}\right]+\left[L^{c}, D^{c} L^{c}\right] \subset\left[L^{c}, L^{c}\right] .
$$

Ainsi la restriction à $L$ d'une dérivation $D^{c}$ de $L^{c}$ est une dérivation $D=\mathscr{L}(X)$ (où $X \in L^{c}$ ) de $L$.

Nous allons montrer que, sous l'hypothèse faite, toute dérivation de $L^{c}$ est intérieure. Si $Y \in L^{c}, Z \in L$ on a avec les notations précédenets:

$$
D^{c}[Y, Z]=\left[D^{c} Y, Z\right]+\left[Y, D^{c} Z\right]
$$


soit:

$$
\mathscr{L}(X)[Y, Z]=\left[D^{c} Y, Z\right]+[Y, \mathscr{L}(X) Z] .
$$

Or, $\mathscr{L}(X)$ définissant une dérivation de $L^{c}$ (Jacobi) :

$$
\mathscr{L}(X)[Y, Z]=[\mathscr{L}(X) Y, Z]+[Y, \mathscr{L}(X) Z]
$$

Il en résulte que pour tout $Z \in L$ :

$$
\left[\left(D^{c}-\mathscr{L}(X) Y, Z\right]=0 .\right.
$$

$\left(D^{c}-\mathscr{L}(X)\right) Y$ est un élément de $L^{c}$ correspondant à une constante $K$. Prenons pour $Z$ un élément de $L_{0}^{*}$; on a $\mu(Z)=d v$, où $v \in N_{0}$. D'après (5.3), (18.3) s'écrit :

$$
d \Lambda\left\{\mu\left(D^{c} Y-\mathscr{L}(X) Y\right) \wedge d v\right\}+K d v=0
$$

soit:

$$
\Lambda\left\{\mu\left(D^{c} Y-\mathscr{L}(X) Y\right) \wedge d v\right\}+K v=C=\text { const. }
$$

Le premier membre étant à support compact, $C=0$ et pour tout $v \in N_{0}$

$$
\Lambda\left\{\mu\left(D^{c} Y-\mathscr{L}(X) Y\right) \wedge d v\right\}+K v=0 .
$$

$x$ étant un point de $\mathrm{W}$, choisissons $v \in N_{0}$ de façon que $v$ s'annule en $x$ et que $(d v)_{x}$ soit égal à un covecteur arbitraire donné. On déduit de (18.4) que $\left\{D^{c} Y-\mathscr{L}(X) Y\right\} Y_{x}=0$. Par suite pour tout $Y \in L^{c}$

$$
D^{c} Y=\mathscr{L}(X) Y \quad\left(X \in L^{c}\right),
$$

et $D^{c}$ est une dérivation intérieure.

Si $F$ n'est pas exacte, toute dérivation de $L=L^{c}$ est aussi intérieure d'après le théorème 1.

Théorème 2. Toute dérivation de l'algèbre de Lie $L^{c}$ est intérieure.

\section{Cohomologie de $L, L^{*}$ et $L^{c}$}

Les $p$-cochaines $b$ de $L$ sont les applications $p$-linéaires alternées de $L^{p}$ dans $L$, les $o$-cochaînes s'identifiant à des éléments de $L$. La différentielle de la $p$-cochaîne $b$ est la $(p+1)$-cochaîne $d b$ définie par

$$
\begin{aligned}
d b\left(X_{0}, \cdots, X_{p}\right)= & \sum_{k=0}^{p}(-1)^{k}\left[X_{k}, b\left(X_{0}, \cdots, \hat{X}_{k}, \cdots, X_{p}\right)\right] \\
& +\sum_{k<l}(-1)^{k+l} b\left(\left[X_{k}, X_{l}\right], X_{0}, \cdots, \hat{X}_{k}, \cdots, \hat{X}_{l}, \cdots, X_{p}\right),
\end{aligned}
$$


où $X_{0}, \cdots, X_{p} \in L$ et où ^ désigne l'omission. Si $b=d Z$, où $Z \in L$, on a $b(X)=[X, Z]=[-Z, X]$. Si $b$ est une 1-cochaîne

$$
d b(Y, Z)=[Y, b(Z)]+[b(Y), Z]-b([Y, Z]) \quad(Y, Z \in L) .
$$

L'espace des 1-cochaînes fermées de $L$ coïncide avec l'espace des dérivations de $L$, celui des 1-cochaînes exactes avec celui des dérivations intérieures. D'après le théorème $1 \mathrm{du} \S 18$, ces deux espaces sont isomorphes respectivement a $L^{c}$ et $L$; leur quotient est l'espace de cohomologie $H^{1}(L ; L)$. Le même raisonnement montre que $H^{1}\left(L^{*} ; L^{*}\right)$ est isomorphe à l'espace $L^{c} / L^{*}$ et que $H^{1}\left(L^{c} ; L^{c}\right)=0$.

Nous avons vu d'autre part (proposition $2 \mathrm{du} \S 3$ ) que l'espace de cohomologie $H^{1}(W ; R)$ à supports fermés est isomorphe à $L / L^{*}$ et que si $F$ est exacte, $\operatorname{dim} L^{c} / L=1$ (§5). On en déduit

Théorème. L'espace de cohomologie $H^{1}(L ; L)$ est nul. L'espace de cohomologie $H^{1}(L ; L)$ est isomorphe à $L^{c} / L$, l'espace de cohomologie $H^{1}\left(L^{*} ; L^{*}\right)$ $\grave{a} L^{c} / L^{*}$. Si F n'est pas exacte (en particulier si $W$ est compacte) $\operatorname{dim} H^{1}(L ; L)$ $=0, \operatorname{dim} H^{1}\left(L^{*} ; L^{*}\right)=b_{1}(W) ;$ si $F$ est exacte $\operatorname{dim} H^{1}(L ; L)=1, \operatorname{dim}$ $H^{1}\left(L^{*} ; L^{*}\right)=b_{1}(W)+1$, où $b_{1}(W)$ est le premier nombre de Betti de $W$ pour l'homologie à supports compacts.

\section{Dérivations de $N$}

a) Supposons $W$ non compacte; soit $\mathscr{D}$ une dérivation de $N$. Si $u \in N$, il existe une $(2 n-1)$-forme $\psi$ de $W$ telle que $u \eta=d \psi$.

Introduisons un recouvrement $\left\{U_{\nu}\right\}_{\nu \in I}$ par des domaines contractiles d'un voisinage ouvert $E$ de $S(\psi)$ avec la condition suivante (voir $\S 12$, a): il existe une partition de $I$ en une collection finie de sous ensembles $I_{\mu}(\mu=1, \cdots, r)$ telle que, pour chaque $\mu$, les domaines pour lesquelles $\nu \in I_{\mu}$ soient deux à deux disjoints. Soit $\left\{\varphi_{\nu}\right\}$ une partition de l'unité subordonnée et posons $\tau_{\mu}=\sum_{I_{\mu}} \varphi_{\nu}$, $\psi_{\mu}=\tau_{\mu} \psi, u_{\mu} \eta=d \psi_{\mu}$. Pour $\mu$ fixé, considérons les domaines $\left\{U_{\nu}\right\}_{\nu \in I_{\mu}}$ deux à deux disjoints; en appliquant à $\left.u_{\mu}\right|_{U_{\nu}}$ le lemme de Calabi, on voit que $u_{\mu}$ est la somme des parenthèses de $2 n$ couples $\left(v_{(i)}, w^{(i)}\right)$ d'éléments de $N$, où $S\left(v_{(i)}\right)$, $S\left(w^{(i)}\right) \subset U_{I_{\mu}} U_{\nu}$; par suite $S\left(\mathscr{D} u_{\mu}\right) \subset U_{I_{\mu}} U_{\nu}$ et $S(\mathscr{D} u) \subset E$. Ainsi $S(\mathscr{D} u) \subset S(\psi)$. On en déduit comme pour $\mathscr{D}_{1}(\S 16, \mathrm{c})$ que $\mathscr{D}$ est locale.

L' espace des dérivations de $N$ est isomorphe à $L^{c}$, celui des dérivations intérieures à $L^{*}$. Ainsi

Théorème 1. Si $W$ est non compacte, toute dérivation de l'algèbre de Lie $N$ est locale et est donnée par $\mathscr{L}(X)+K_{X}$, où $X \in L^{c}$. L'espace de cohomologie $H^{1}(N ; N)$ de l'algèbre $N$ est isomorphe à l'espace $L_{c} / L^{*}$ et a même dimension que $H^{1}\left(L^{*} ; L^{*}\right)$.

b) Supposons $W$ compacte. Tout élément $u$ de $N$ admet la décomposition unique 


$$
u=u_{1}+V^{-1} \int_{W} u \eta
$$

où $u_{1} \in N_{1}$ et $V$ est le volume de $W$. Si $\mathscr{D}$ est une dérivation de $N$, sa restriction à $N_{1}$ est une dérivation $\mathscr{L}(X)$ de $N_{1}$, où $X \in L$. On a :

$$
\mathscr{D} u_{1}=\mathscr{L}(X) u_{1}=\mathscr{L}(X) u \text {. }
$$

Il en résulte qu'à toute dérivation $\mathscr{D}$ de $N$ on peut associer un élément $X$ de $L$ et une constante $\mathscr{D} 1$ telle que $\mathscr{D}$ s'écrive

$$
\mathscr{D} u=\mathscr{L}(X) u+V^{-1} \mathscr{D} 1 \cdot \int_{W} u \eta
$$

Pour $\mathscr{D} 1 \neq 0, \mathscr{D}$ est une dérivation non locale.

Théorème 2. Si $W$ est compacte, toute dérivation de l'algèbre de Lie $N$ est donnée par:

$$
\mathscr{D} u=\mathscr{L}(X) u+V^{-1} \mathscr{D} 1 \cdot \int_{W} u \eta
$$

où $X \in L$ et $\mathscr{D} 1 \in R$. On a dim $H^{1}(N ; R)=b_{1}(W)+1$.

c) Si $W$ est non compacte, la "cohomologie à supports compacts" de $N$ peut être défini par les cochaînes de $N$ à valeurs dans l'idéal $N_{0}$, les 0 -chaînes s'identifiant aux éléments de $N_{0}$. Une telle cochaîne fermée est une dérivation $\mathscr{D}$ de $N$ telle que pour tout $u \in N, \mathscr{D} u$ soit à support compact. En particulier $\mathscr{D} 1$ devant être à support compact, on a $\mathscr{D} 1=0$. Par suite la dérivation $\mathscr{D}$ de $N$ envisagée est nécessairement de la forme $\mathscr{D}=\mathscr{L}(X)$, où $X \in L$.

Montrons que $S(X)$ est compact. S'il n'en est pas ainsi, il existe une suite $\left\{x_{\nu}\right\}$ de points de $W$ tels que $X\left(x_{\nu}\right) \neq 0$ et qui n'admet pas de point d'accumulation. Introduisons des pavés ouverts $P_{\nu}$ deux à deux disjoints, arbitrairement petits, de centres $x_{\nu}$. A chaque $P_{\nu}$ on peut faire correspondre une fonction $u_{\nu} \in N$ à support dans $P_{\nu}$, telle que $\mathscr{L}(X) u_{\nu}$ soit $\neq 0$ en $x_{\nu}$. La fonction $u=$ $\sum u_{\nu} \in N$ est telle que $\mathscr{L}(X) u$ est différente de 0 aux points $x_{\nu}$ de la suite et ne peut être à support compact. Ainsi $X \in L_{0}$.

L'espace des 1-cochaînes fermées à valeurs dans $N_{0}$ est donc isomorphe à $L_{0}$ et celui des 1-cochaînes exactes à $L_{0}^{*}$. Leur quotient est l'espace de cohomologie ${ }_{0} H^{1}(N ; N)$ à supports compacts isomorphe à $L_{0} / L_{0}^{*}$. Sa dimension est le premier nombre de Betti $b_{1}^{0}(W)$ pour l'homologie à supports fermés.

\section{Bibliographie}

[1] A. Avez, Cas de réductivité d'algèbres de Lie de symplectomorphisms, C. R. Acad. Sci. Paris 274 (1972) 1729-1733.

[2] A. Avez \& A. Lichnerowicz, Dérivations et premier groupe de cohomologie pour des algèbres de Lie attachées à une variété symplectique, C. R. Acad. Sci. Paris 275 (1972) 113-118. 
[ 3 ] E. Calabi, On the group of automorphisms of a symplectic manifold, Problems in Analysis, A Sympos. in Honor of S. Bochner, Princeton University Press, Princeton, 1970, 1-26.

[4] E. Cartan, Les sous-groupes des groupes continus de transformations, Ann. Sci. École Norm. Sup. 25 (1908) 57-194; Leçons sur les invariants intégraux, Hermann, Paris, 1922.

[5] C. Chevalley \& S. Eilenberg, Cohomology theory of Lie groups and Lie algebras, Trans. Amer. Math. Soc. 63 (1948) 85-124.

[6] A. Diaz-Miranda, Idéaux de l'algèbre de Lie des champs de vecteurs hamiltoniens, C. R. Acad. Sci. Paris 274 (1972) 989-992.

[ 7 ] P. Liebermann, Sur les automorphismes infinitesimaux des structures symplectiques et des structures de contact, Colloq. Géometrie Differentielle Globale (Bruxelles 1958), Louvain, 1959, 37-59.

[ 8 ] A. Lichnerowicz, Sur les variétés symplectiques, C. R. Acad. Sci. Paris 233 (1951) 723-726.

[9] _- Sur la réductivité de certaines algèbres d'automorphismes, C. R. Acad. Sci. Paris 253 (1961) 1302-1304; Théorème de réductivité sur des algèbres d'automorphismes, Rend. Mat. 22 (1962) 197-244.

[10] _- Sur l'algèbre de Lie des automorphismes symplectiques, C. R. Acad. Sci. Paris 274 (1972) 1177-1180.

[11] J. H. C. Whitehead, On the decomposition of an infinitesimal group, Proc. Cambridge Philos. Soc. 32 (1936) 229-237.

[12] V. I Arnold, One-dimensional cohomologies of Lie algebras of nondivergent vector fields and rotation numbers of dynamic systems, Functional Anal. Appl. 3 (1969) 319-321.

Université de Paris VI

Collège de France

Université de PARIS VI 